\title{
Load Spectrum and Fatigue Life Computer Analysis of Prestressed Concrete Bridges
}

\author{
Xin Qin ${ }^{1,2}$, Dou Yuanming ${ }^{1,2}$ and Chen Wan ${ }^{1,2}$ \\ ${ }^{1}$ School of Civil Engineering, Hebei University of Technology, No. 5340 Xi-ping \\ Street, Beichen District, Tianjin, China, 300401; \\ ${ }^{2}$ Civil Engineering Technology Research Center of Hebei Province, No.5340 Xi- \\ ping Street, Beichen District, Tianjin, China, 300401 \\ douyuanming2014@126.com
}

\begin{abstract}
With respect to fatigue damage of heavy traffic on the bridge structure, this paper studied on load spectrum and fatigue simulation. The author used manual recording, live cameras, vehicle dynamic weighing system (WIM) to conduct traffic investigation in a continuous 24-hour period. Basing on these data in Hebei province, we established typical vehicle load spectrum using probabilistic and statistical methods, and deduced a standard fatigue vehicle model on this basis. Then we prepared random load spectrum by $M A T L A B$ and realized loading it on a finite element model (FEM). Next, the paper assessed the fatigue life of in-service highway bridges that in Hebei by comparing typical vehicle load and random vehicle load. Results showed that vehicle speed has significant influence on fatigue damage under typical vehicle load. Equivalent stress amplitude is small in two operational states under random vehicle load, the general operation state and intensive operation state. Fatigue damage to bridge under general operation is obvious. After verified the applicability of the standard fatigue vehicle model, we can believe that this model developed in this article can be further used as a reference to check highway fatigue damage in the entire Hebei province. Moreover, all the results showed that the bridges wouldn't get fatigue failure under a normal operation state when there is no impact of environmental factors.
\end{abstract}

Keywords: Heavy-duty traffic; Random traffic flow; Fatigue damage; Finite element modeling

\section{Introduction}

With rapid development of transportation, fatigue damage of bridges under heavy-duty traffic occurs frequently. The actual service load at present has far exceeded the designed load of bridges at the time they were constructed. So it is necessary to study fatigue damage of old highway bridges under current vehicle load [1-2]. So far bridge fatigue load has been studied a lot in countries such as the United States, the UK and Japan [3-5] etc.

Standard vehicle load spectrum and fatigue vehicle model, which are based on domestic traffic situation [6-9], are contained in their bridge design specifications. UK BS5400 specification provides a good example. However, our region, Hebei province, has not established any vehicle load spectrum that suits for highway and city road bridge fatigue design. Although Tong Lewei [10], Zhou Yongtao [11] and Zhu Hongbing [12] etc. have carried out relevant research on load spectrum and fatigue vehicle model respectively, their research only adapt to local traffic, which cannot can reflect the national situation. 
In this paper, manual recording, live cameras, vehicle dynamic weighing system (WIM) was used to conduct the traffic investigation in a continuous 24-hour period. By combining on-the-spot traffic survey data, we built a separating entity finite element model (FEM) about a 16-meter prestressed reinforced concrete hollow slab girder via finite element analysis software [13-14] and realized loading random traffic flow produced by MATLAB on this model [15-16].

\section{Modeling}

\subsection{Typical Vehicle Modeling}

Current specifications of China divide vehicle load of urban bridges into urbanlevel A and urban level-B as well as highway bridges into road-level I road-level II, which does not reflect the actual highway bridges load operations. By referring to domestic and foreign research achievements ${ }^{[17-19]}$, our research group selected highway sectionsG4 and G45 of Hebei in November 2013, carried out traffic surveys and axle load statistics. We used the principle of fatigue damage equivalence, obtained corresponding typical vehicle load spectrum (Table 1).

Table 1. Typical Vehicle Load Spectrum

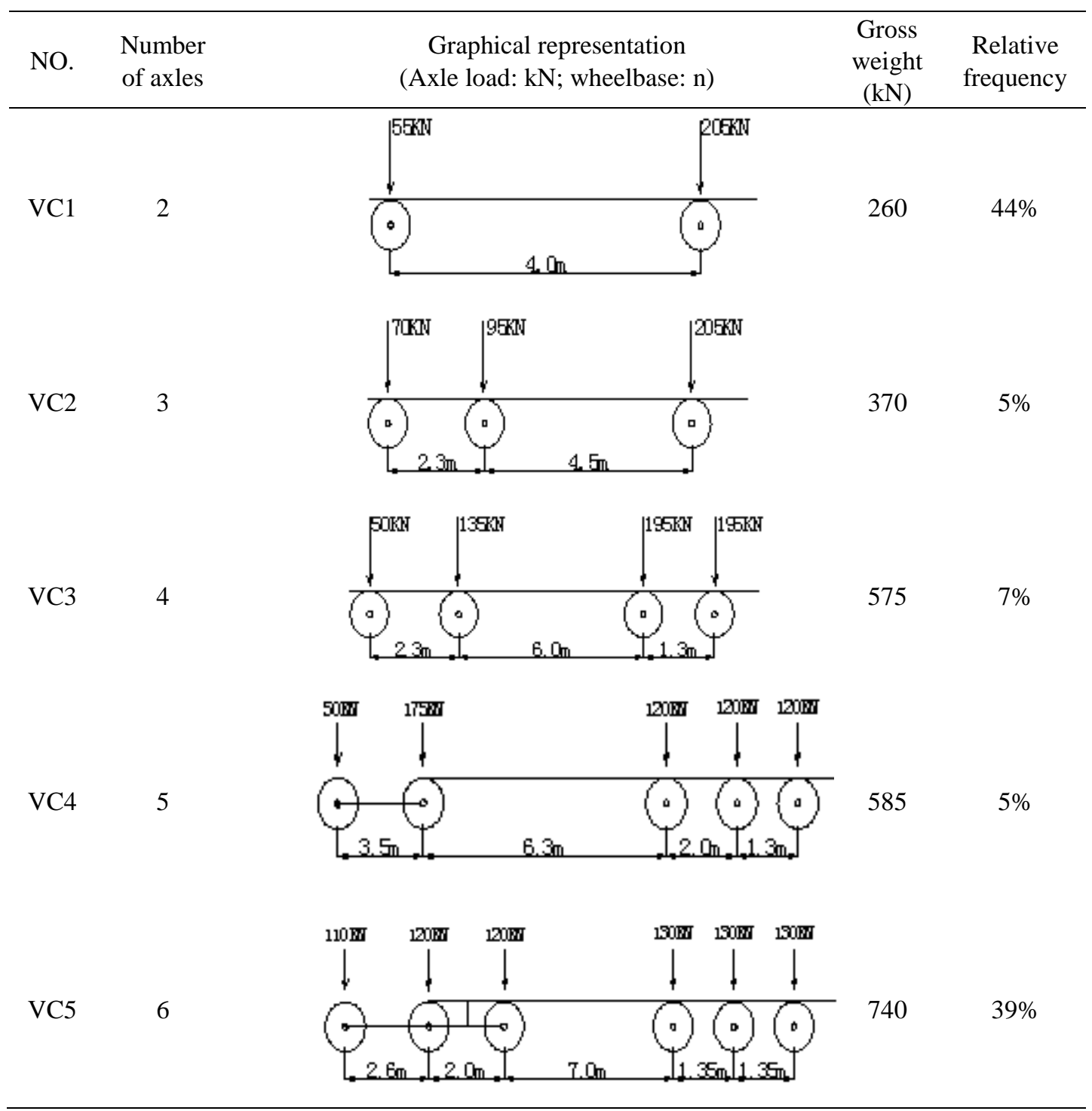


Many fatigue computations show that the fatigue damage brought about by 6 -axle vehicles for bridge is most common. Thus, we mainly use 6 -axle vehicles as a prototype to deduce corresponding standard fatigue vehicle models. By consulting the way to establish standard fatigue vehicle models about highway bridges in southwestern mountainous areas ${ }^{[20]}$, the research group built a standard fatigue vehicle model about highway bridges in the section based on VC5 vehicle type in Table 1 and kept wheelbase the same as that of VC5 vehicle type.

According to $W=\left(\sum f_{i} W_{i}^{3}\right)^{1 / 3}$, gross weight of a standard fatigue vehicle is $\mathrm{W}=575 \mathrm{KN}$. By distributing the weight to each axle of a 6 -axle vehicle in proportion, a schematic diagram about the standard fatigue vehicle model was got, as showed in Fig. 1.

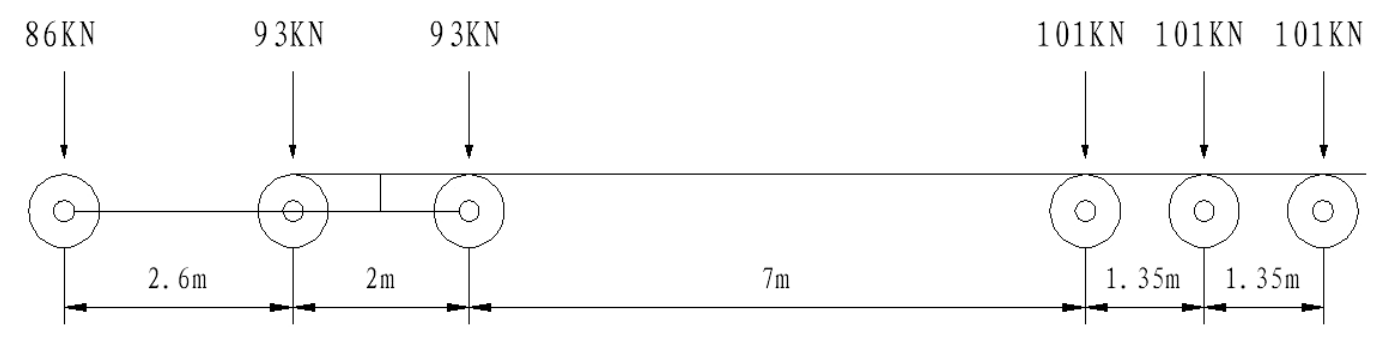

Figure 1. A Schematic Diagram of Standard Fatigue Vehicle Models on Highway Bridges

There $f_{i}$ is the frequency in which the $i$ th type of typical vehicles appears and $W_{i}$ denotes gross weight of the $i$ th type of typical vehicles.

\subsection{Compiling Random Load Spectrum}

Representative vehicle distribution concluded by related investigation and statistics in China is shown in Table 2 [21].

Table 2. Distribution Law about Traffic Flow and Load Statistics

\begin{tabular}{|c|c|c|c|c|c|}
\hline \multirow{2}{*}{\multicolumn{2}{|c|}{$\frac{\text { Random vehicles }}{\text { Gross vehicle weight (t) }}$}} & \multirow{2}{*}{$\frac{\text { Capacity }}{12462}$} & \multirow{2}{*}{$\begin{array}{c}\text { Distribution } \\
\text { law }\end{array}$} & \multicolumn{2}{|c|}{ Distribution parameter } \\
\hline & & & & $\hat{\mu}=1.666697$ & $\hat{\sigma}=0.816272$ \\
\hline \multicolumn{2}{|c|}{ Axle load (t) } & 31836 & Lognormal & $\hat{\mu}=0.633925$ & $\hat{\sigma}=0.922265$ \\
\hline \multirow{2}{*}{$\begin{array}{l}\text { Distance } \\
\text { headway } \\
\quad(\mathrm{m})\end{array}$} & $\begin{array}{c}\text { General operation } \\
\text { state }\end{array}$ & 12462 & Lognormal & $\hat{\mu}=4.827692$ & $\hat{\sigma}=1.115751$ \\
\hline & $\begin{array}{c}\text { Intensive } \\
\text { operation state }\end{array}$ & 389 & Lognormal & $\hat{\mu}=1.561165$ & $\hat{\sigma}=0.279707$ \\
\hline \multirow{2}{*}{$\begin{array}{l}\text { Time } \\
\text { interval } \\
(\mathrm{s})\end{array}$} & $\begin{array}{l}\text { General operation } \\
\text { state }\end{array}$ & 12462 & Gamma & $\hat{\alpha}=0.904286$ & $\hat{\lambda}=0.039451$ \\
\hline & $\begin{array}{c}\text { Intensive } \\
\text { operation state }\end{array}$ & 389 & Gamma & $\hat{\alpha}=12.907330$ & $\hat{\lambda}=7.235810$ \\
\hline
\end{tabular}

Distance headway distribution obtained by traffic flow statistics for a highway under a general operation state is illustrated in Figure 2. 


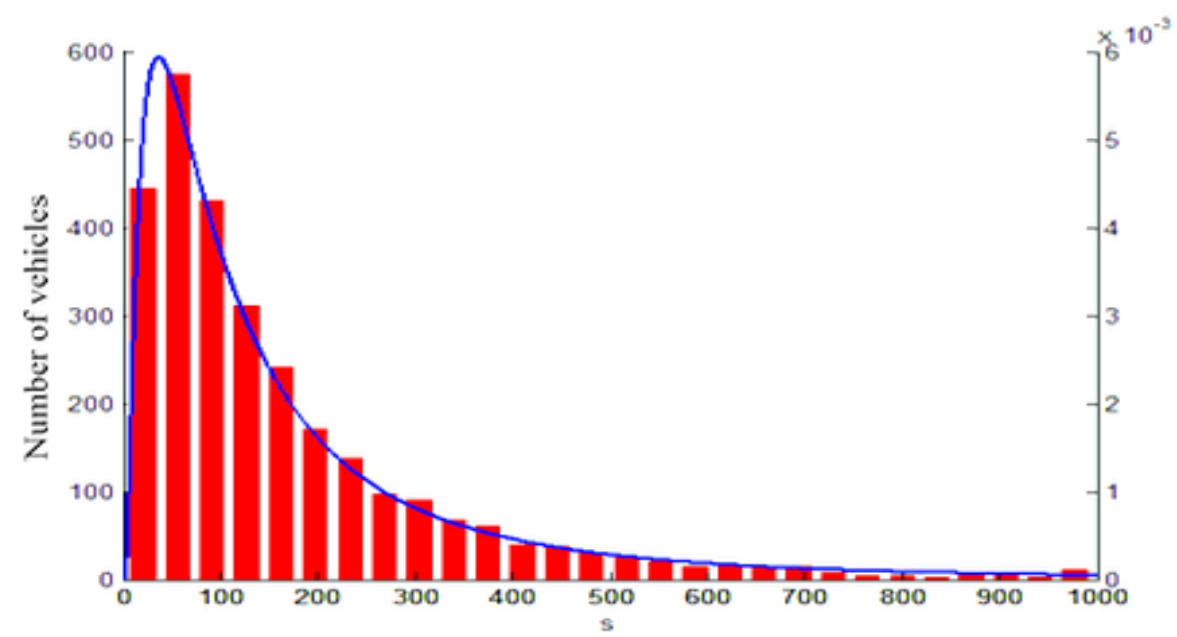

Figure 2. A Histogram about Distribution of Distance Headway under a General Operation State

According to distribution fitting, the distance headway under a general operation state obeys the log-normal distribution with parameters $\mu=4.7115$ and $\sigma=1.0702$. By comparing parameters in Table 1, the author has concluded that the distribution of distance headway is closer. Thus, $\mu=1.561165$ and $\sigma=0.279707$ were selected under an intensive operation state of vehicles. Random distance headway was simulated by MATLAB according to these two distributions, as showed in Figure 3. The random traffic flow-generating program compiled by MATLAB makes vehicle load equivalent to axle load in the form of concentrated force. Then, we exported data files in $t x t$, which prepares for the situation of loading random vehicle load on a bridge structure using finite element model (FEM) [2223].

The procedures of the MATLAB program are as follows:

a. According to the data, generate $\mathrm{N}$ random vehicle type and $\mathrm{N}$ random distance headway.

b. For each typical vehicle axle and wheelbase is identified, we assign the corresponding load to each axis.

c. Record the initial position of the random traffic each axis on the bridge. Then output axle load in the corresponding position.

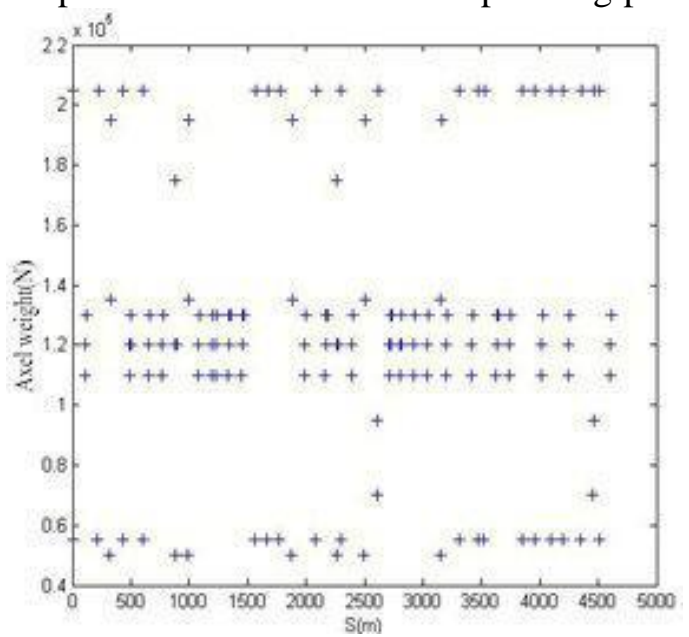

(a) A general operation state

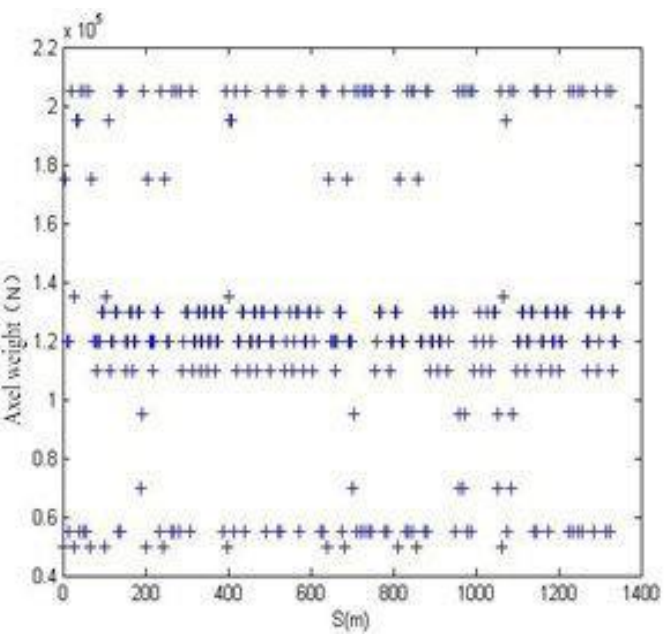

(b) A general operation state

Figure 3. Distribution of Random Axle Weight and Location 


\section{Fem Establishment}

To establish a FEM of prestressed reinforced concrete bridges, the author selected a 16-meter simply supported prestressed hollow slab bridge on the highway. Besides, the author used Solid65 to simulate concrete and Link8 to steel and prestressed reinforcement, and utilized falling temperature method to realize pres-tress application. Figure 4-5 displays bridge design data. Considering wheelbase features of model vehicles, simple support constraint was implemented to beam-ends in order that random vehicle axle load could be applied conveniently. Figure 6 shows unit division methods.

In order to realize loading of MATLAB random traffic flow, APDL language was used to program JIAZAI. As can be seen from the load simulation that MATLAB program may generate random vehicles that comply with designated situations and demands, and make adjustments based on vehicle types in different regions. Thus this program gets a nice versatility.

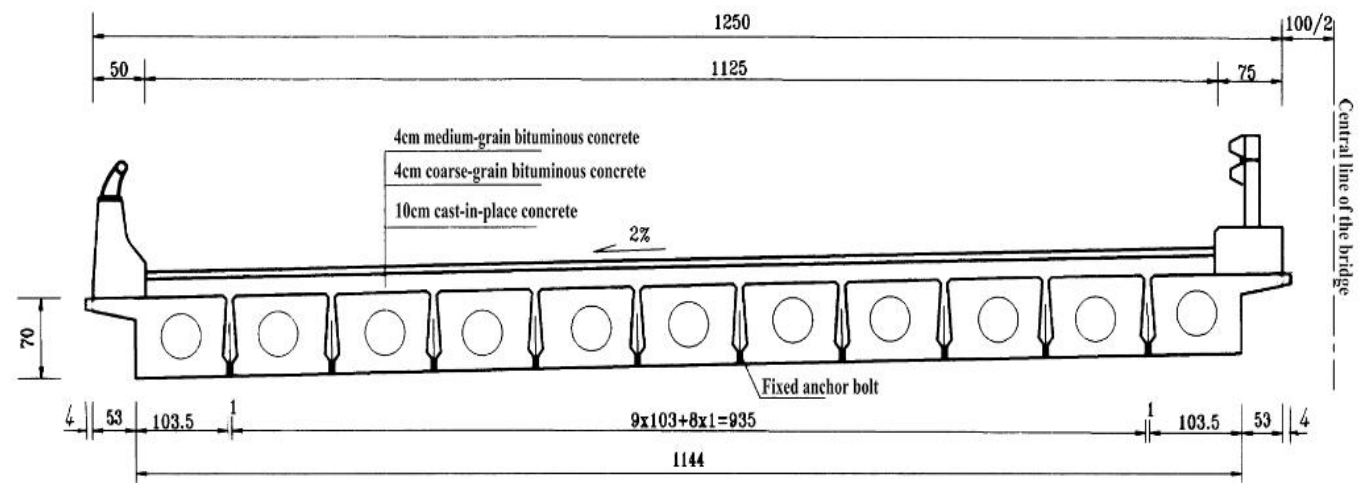

Figure 4. A Cross Section of a Hollow Slab Bridge
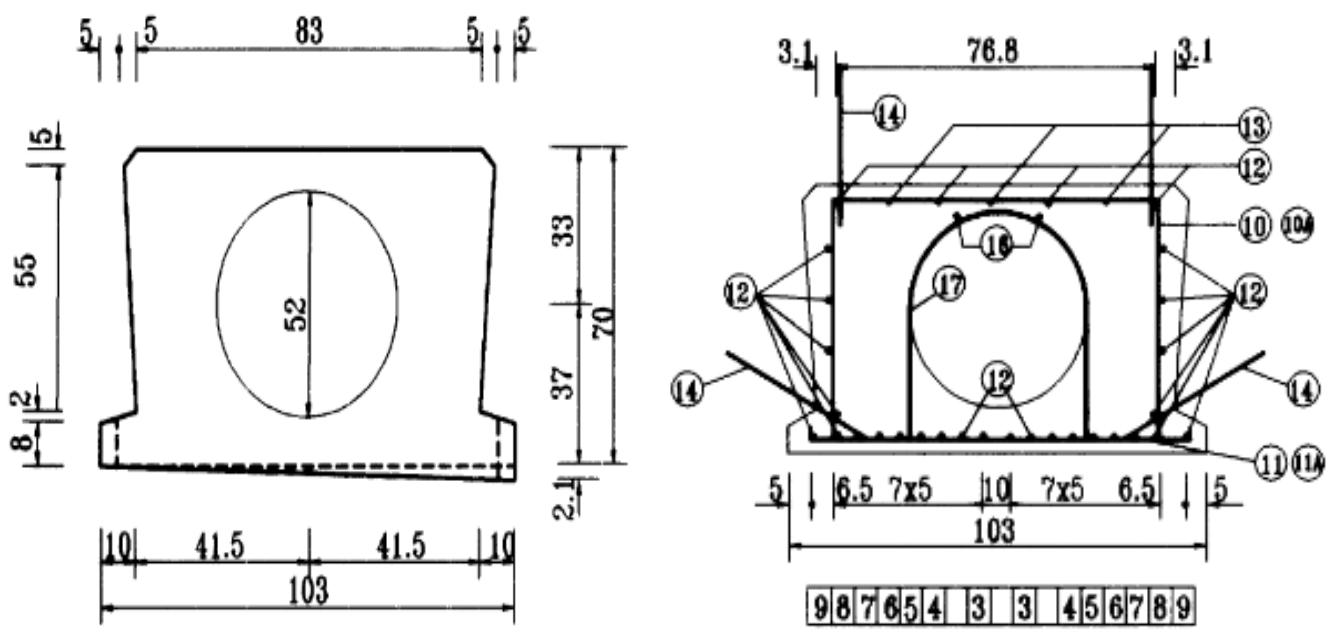

Figure 5. Sectional Dimension of a Hollow Slab and Reinforcement Drawing 

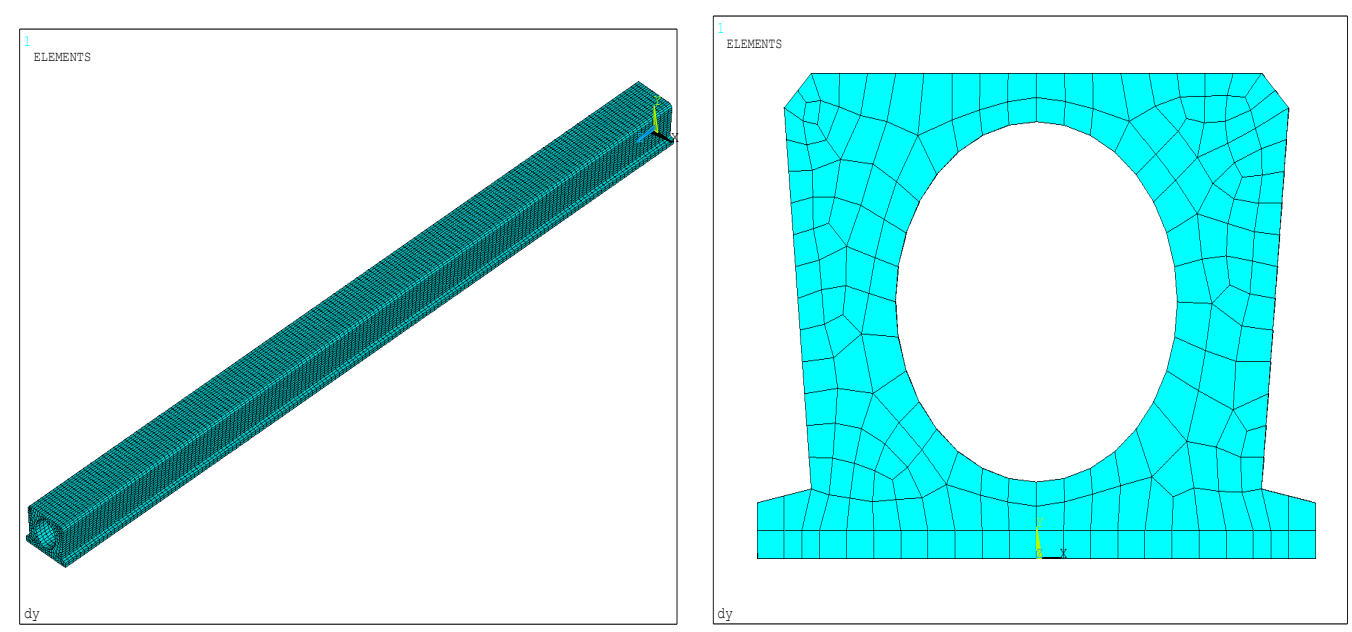

Figure 6. FEM Mesh Generation

\section{Fatigue Damage Analysis}

\subsection{Typical Vehicle Modeling}

Depending on Table 1, the author divided each model vehicle into 6 working conditions (Table 3) and defined two variables including vehicle speed and distance headway. In the process of loading, we also considered motor vehicle loading coefficient and influence line coefficient of lateral distribution.

4.1.1 Dynamic Response of Girders: The author applied the foregoing working conditions to FEM, carried out transient dynamic analysis, extracted stress time-history data about midspan concrete and prestressed reinforcement under each working condition, performed counting by rain-flow counting method and obtained corresponding stress amplitude and cycle index. As a 2-axle vehicle and 6-axle ones account for $83 \%$ of typical vehicle load, only time-history curves for midspan nodes of the two types of vehicles under different working conditions are illustrated here, as showed in Fig. 7-10.

Table 3. Working Conditions of Typical Vehicle Loading

\begin{tabular}{|c|c|c|c|c|c|c|}
\hline \multirow{2}{*}{$\begin{array}{l}\text { Working } \\
\text { condition }\end{array}$} & Condition 1 & Condition 2 & Condition 3 & $\begin{array}{l}\text { Condition } \\
4\end{array}$ & Condition 5 & Condition 6 \\
\hline & \multicolumn{3}{|c|}{$\mathrm{s}=20 \mathrm{~m}$} & \multicolumn{3}{|c|}{$\mathrm{v}=60 \mathrm{~km} / \mathrm{h}$} \\
\hline $\mathrm{VC} 1$ & $\mathrm{v}=20 \mathrm{~km} / \mathrm{h}$ & $\mathrm{v}=40 \mathrm{~km} / \mathrm{h}$ & $\mathrm{v}=80 \mathrm{~km} / \mathrm{h}$ & $\mathrm{s}=10 \mathrm{~m}$ & $\mathrm{~s}=20 \mathrm{~m}$ & $\mathrm{~s}=40 \mathrm{~m}$ \\
\hline $\mathrm{VC} 2$ & $\mathrm{v}=20 \mathrm{~km} / \mathrm{h}$ & $\mathrm{v}=40 \mathrm{~km} / \mathrm{h}$ & $\mathrm{v}=80 \mathrm{~km} / \mathrm{h}$ & $\mathrm{s}=10 \mathrm{~m}$ & $\mathrm{~s}=20 \mathrm{~m}$ & $\mathrm{~s}=40 \mathrm{~m}$ \\
\hline $\mathrm{VC} 3$ & $\mathrm{v}=20 \mathrm{~km} / \mathrm{h}$ & $\mathrm{v}=40 \mathrm{~km} / \mathrm{h}$ & $\mathrm{v}=80 \mathrm{~km} / \mathrm{h}$ & $\mathrm{s}=10 \mathrm{~m}$ & $\mathrm{~s}=20 \mathrm{~m}$ & $\mathrm{~s}=40 \mathrm{~m}$ \\
\hline $\mathrm{VC} 4$ & $\mathrm{v}=20 \mathrm{~km} / \mathrm{h}$ & $\mathrm{v}=40 \mathrm{~km} / \mathrm{h}$ & $\mathrm{v}=80 \mathrm{~km} / \mathrm{h}$ & $\mathrm{s}=10 \mathrm{~m}$ & $\mathrm{~s}=20 \mathrm{~m}$ & $\mathrm{~s}=40 \mathrm{~m}$ \\
\hline VC5 & $\mathrm{v}=20 \mathrm{~km} / \mathrm{h}$ & $\mathrm{v}=40 \mathrm{~km} / \mathrm{h}$ & $\mathrm{v}=80 \mathrm{~km} / \mathrm{h}$ & $\mathrm{s}=10 \mathrm{~m}$ & $\mathrm{~s}=20 \mathrm{~m}$ & $\mathrm{~s}=40 \mathrm{~m}$ \\
\hline
\end{tabular}




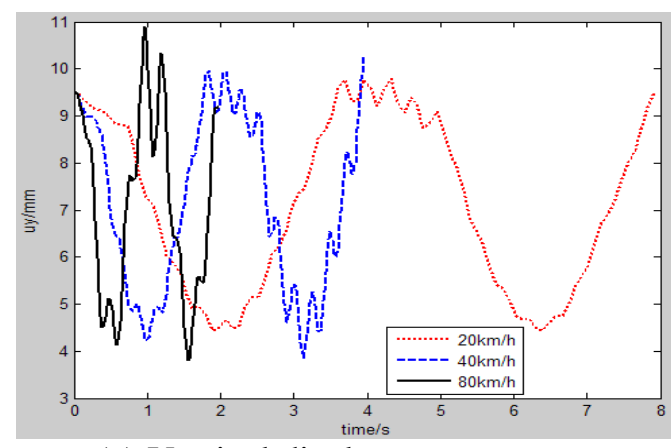

(a) Vertical displacement

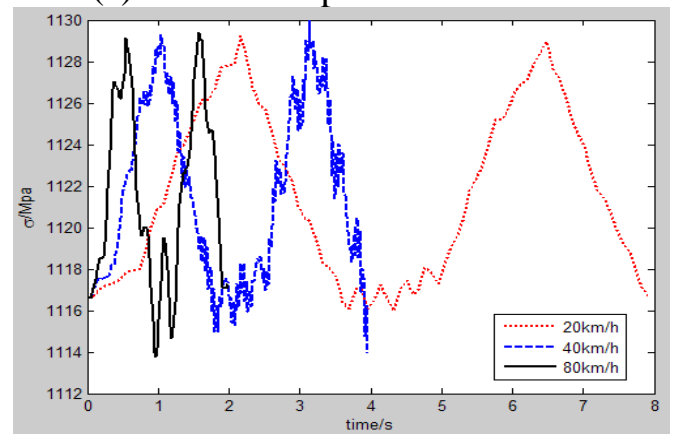

(c) Axial stress of prestressed reinforcement

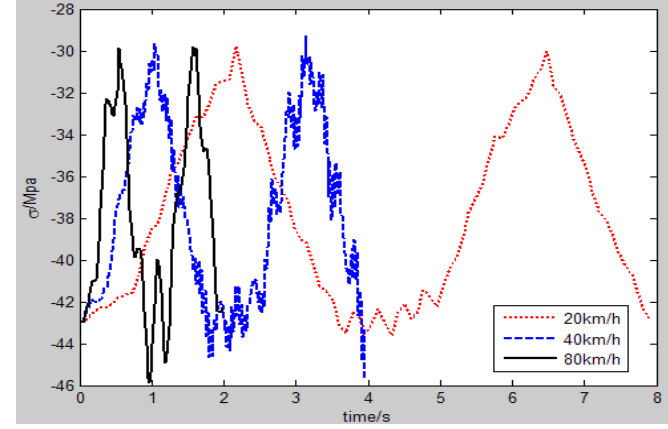

(b) Axial stress of regular reinforcement

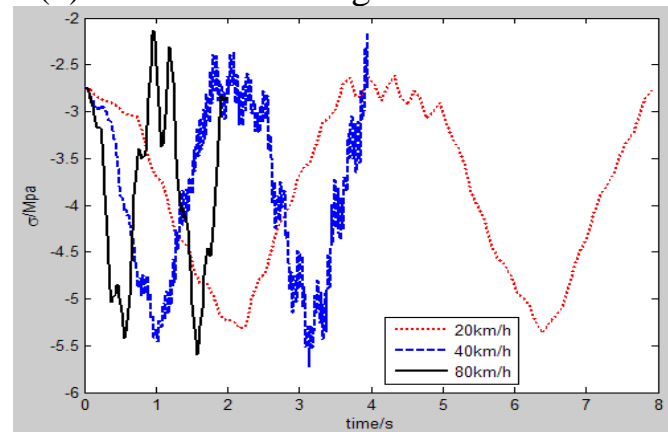

(d) Concrete stress in pressure zones

Figure 7. Time-history Curves about Midspan Nodes of 2-axle Vehicles under Working Conditions 1-3

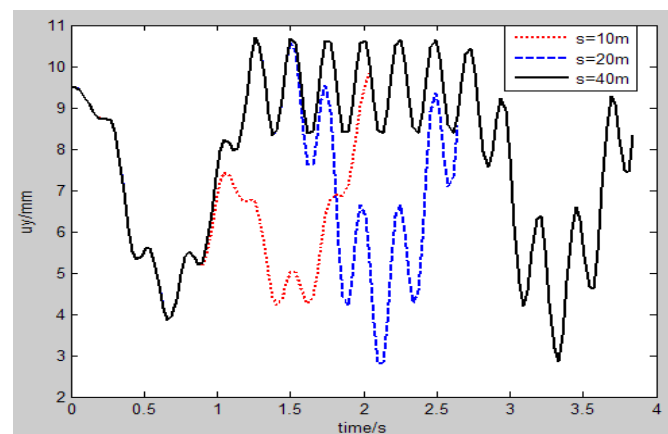

(a) Vertical displacement

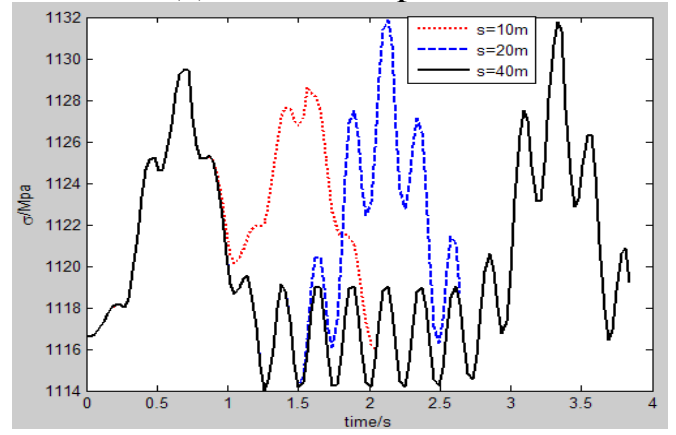

(c) Axial stress of prestressed reinforcement

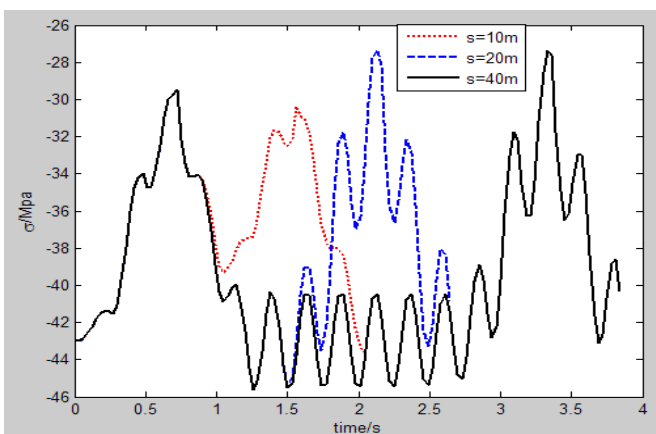

(b) Axial stress of regular reinforcement

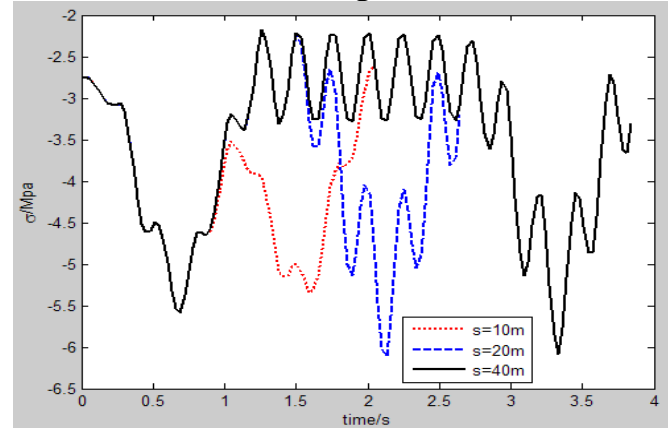

(d) Concrete stress in pressure zones

Figure 8. Time-history Curves about Midspan Nodes of 2-axle Vehicles under Working Conditions 4-6 


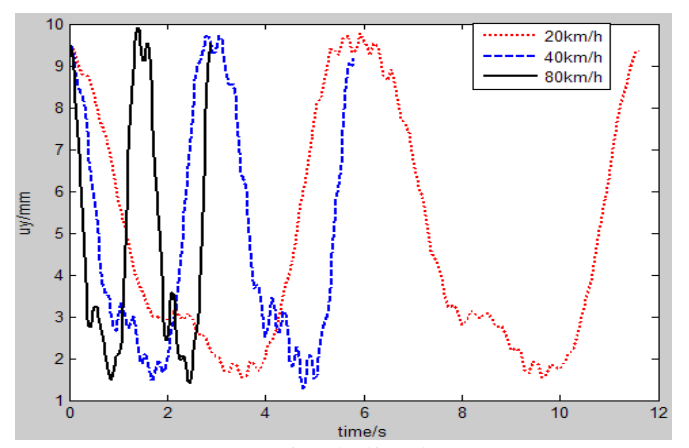

(a) Vertical displacement

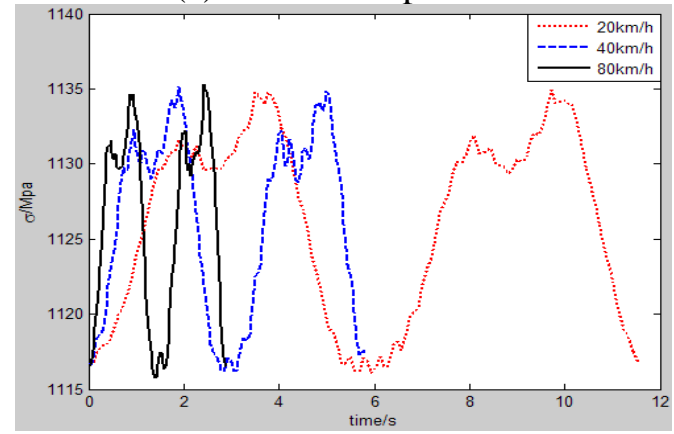

(c) Axial stress of prestressed reinforcement

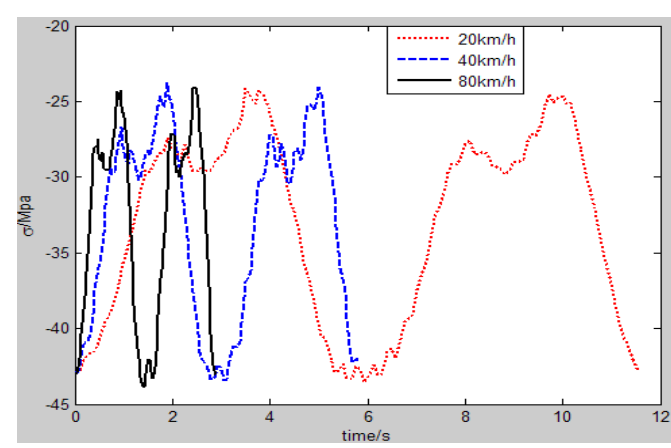

(b) Axial stress of regular reinforcement

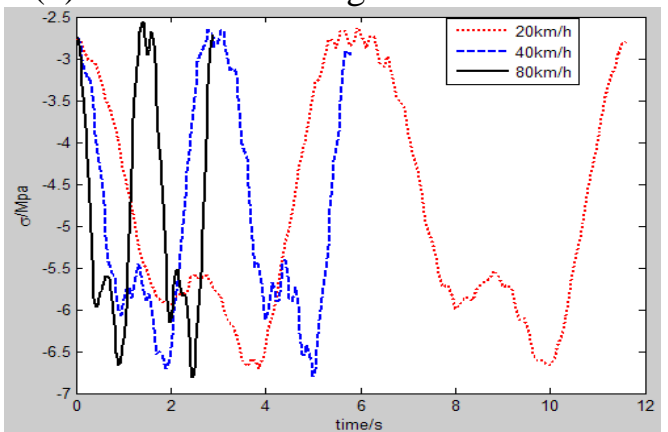

(d) Concrete stress in pressure zones

Figure 9. Time-history Curves about Midspan Nodes of 6-axle Vehicles under Working Conditions 1-3

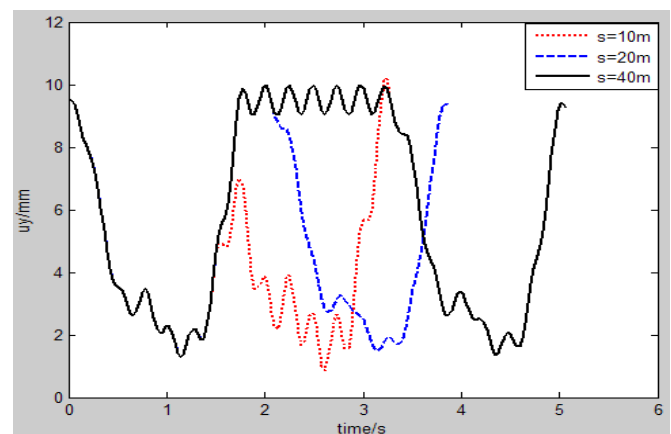

(a) Vertical displacement

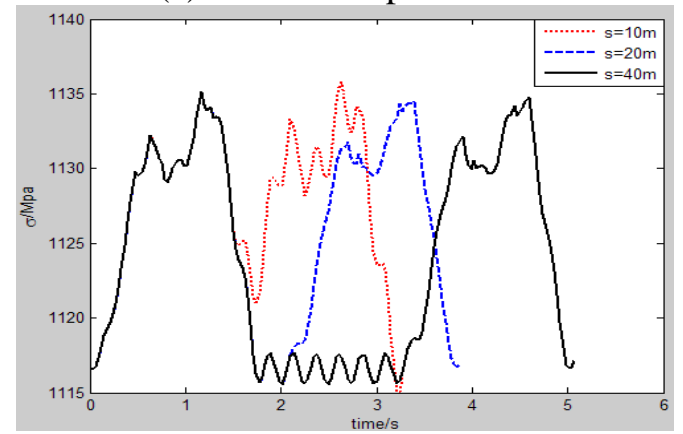

(c) Axial stress of prestressed reinforcement

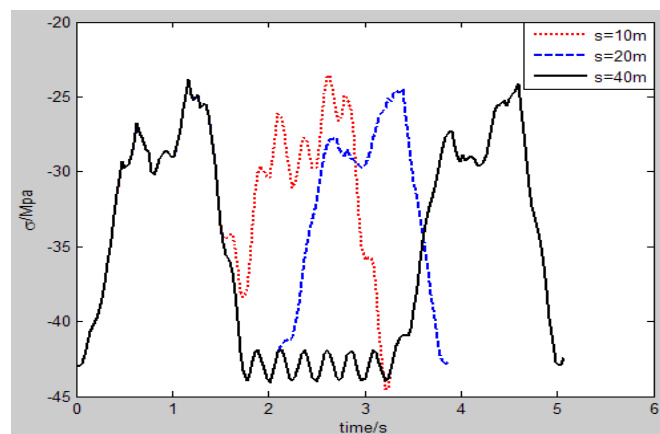

(b) Axial stress of regular reinforcement

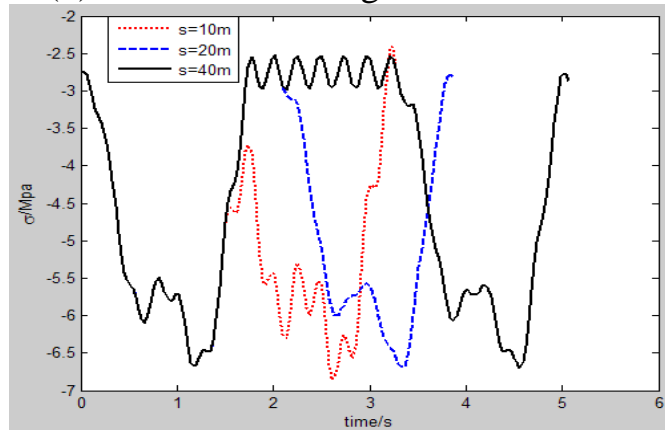

(d) Concrete stress in pressure zones

\section{Figure 10. Time-history Curves about Midspan Nodes of 6-axle Vehicles under Working Conditions 4-6}

Foregoing figures show working conditions 1-3. They indicate that maximum dynamic response and response amplitude of midspan nodes increase with vehicle speed for 2-axle vehicles. Working conditions 4-6 state maximum dynamic response and response amplitude of midspan nodes tend to grow as the distance headway increases. However, 
the trend is not clear. For 6-axle ones, maximum dynamic response and response amplitude of girders under each working condition are approximate.

4.1.2 Calculation about Fatigue Damage: $\mathrm{S}-\mathrm{N}$ curve general formula:

$$
\lg N=A-b \lg S
$$

Under the effect of $n_{i}$ the cycle index of $S_{i}$ random amplitude stress, the damage that structures or components suffer is:

$$
D=\sum \frac{n_{i}}{N_{i}}=\frac{1}{10^{A}} i \sum n_{i}(S)^{b}
$$

The following formula shows fatigue life of structures or components under general stress cycle $S_{\text {eq }}$ :

$$
N=\frac{10^{A}}{\left(S_{e q}\right)^{b}}
$$

Then, under cycle index of equivalent stress level $\sum \mathbf{n}_{\mathbf{i}}$, we may describe structural damage as:

$$
D=\sum \frac{n_{i}}{N_{i}}=\frac{\left(S_{e q}\right)^{m}}{10^{A}} \sum n_{i}
$$

Let Formulas (1.2) and (1.4) be equal. Next, we may deduce the expression of equivalent stress amplitude $S_{\text {eq }}$, i.e.,

$$
S_{e q}=\left[\frac{\sum n_{i}\left(S_{i}\right)^{b}}{\sum n_{i}}\right]^{1 / b}
$$

By consulting experimental study of Li Yongqiang ${ }^{[24-25]}$ et al. S-N rectilinear equation about concrete is:

$$
\begin{cases}\lg S=0.0089-0.0299 \lg N & S \geq 0.78 \\ \lg S=0.0888-0.054 \lg N & S \leq 0.78\end{cases}
$$

From Formula (1.5), the expression related to equivalent stress amplitude $S_{\mathrm{eq}}$ of concrete is:

$$
\left\{\begin{array}{l}
S_{e q}=\left[\frac{\sum n_{i}\left(S_{i}\right)^{33.4448}}{\sum n_{i}}\right]^{0.0299} \\
S_{e q}=\left[\frac{\sum n_{i}\left(S_{i}\right)^{19.8413}}{\sum n_{i}}\right]^{0.0504} \quad S \leq 0.78
\end{array}\right.
$$

By referring to research of China Academy of Railway Sciences on twisted steel and steel strand, $\mathrm{S}-\mathrm{N}$ rectilinear equation of steel is:

$$
\begin{cases}\lg N=15.1348-4.3827 \lg \Delta \sigma & N \leq 10^{7} \\ \lg N=18.8471-6.3827 \lg \Delta \sigma & N \geq 10^{7}\end{cases}
$$

S-N rectilinear equation about prestressed reinforcement is:

$$
\lg N=13.84-3.51 \lg \Delta \sigma
$$

Calculated equivalent stress amplitude of steel $\Delta \sigma_{\text {eq }}$ (unit: MPa): 


$$
\left\{\begin{array}{l}
\Delta \sigma_{e q}=\left[\frac{\sum n_{i}\left(S_{i}\right)^{4.3827}}{\sum n_{i}}\right]^{0.2282} \\
\Delta \sigma_{e q}=\left[\frac{\sum n_{i}\left(S_{i}\right)^{6.3827}}{\sum n_{i}}\right]^{0.1567} \quad N \geq 10^{7} \\
\end{array}\right.
$$

We may describe equivalent stress amplitude of pres-tress $\Delta \sigma_{\text {eq }}$ as:

$$
\Delta \sigma_{e q}=\left[\frac{\sum n_{i}\left(S_{i}\right)^{3.511}}{\sum n_{i}}\right]^{0.2848}
$$

According to the foregoing equivalent stress level and equivalent stress range formulas, we used node stress time-displacement dates which were treated by rainflow counting method, got the damage of midspan nodes in the bridge when each typical vehicle drives on an outside lane. Results are listed in Table 4-8.

Table 4. Fatigue Damage of Midspan Location when VC1 Vehicles Drive under Different Working Conditions

\begin{tabular}{cccccccccc}
\hline \multirow{2}{*}{$\begin{array}{c}\text { Working } \\
\text { condition }\end{array}$} & \multicolumn{3}{c}{ Concrete } & \multicolumn{3}{c}{ Reinforcement } & \multicolumn{2}{c}{ Prestressed reinforcement } \\
\cline { 2 - 9 } & $\mathrm{N}$ & $\begin{array}{c}\text { Fatigue } \\
\text { damage }\end{array}$ & $\Delta \sigma_{\mathrm{eq}}$ & $\mathrm{N}$ & $\begin{array}{c}\text { Fatigue } \\
\text { damage }\end{array}$ & $\Delta \sigma_{\mathrm{eq}}$ & $\mathrm{N}$ & $\begin{array}{c}\text { Fatigue } \\
\text { damage }\end{array}$ \\
\hline $\begin{array}{c}\text { Condition } \\
1\end{array}$ & 0.163 & $\begin{array}{c}2.38 \mathrm{e} 1 \\
7\end{array}$ & $1.47 \mathrm{e}-17$ & 10.7 & $1.80 \mathrm{e} 1$ & $2.23 \mathrm{e}-$ & 8.62 & $3.59 \mathrm{e} 10$ & $\begin{array}{c}1.11 \mathrm{e}- \\
10\end{array}$ \\
$\begin{array}{c}\text { Condition } \\
2\end{array}$ & 0.176 & $\begin{array}{c}5.68 \mathrm{e} 1 \\
6\end{array}$ & $6.91 \mathrm{e}-16$ & 8.69 & $\begin{array}{c}7.16 \mathrm{e} 1 \\
2\end{array}$ & $\begin{array}{c}5.06 \mathrm{e}- \\
12\end{array}$ & 5.30 & $1.99 \mathrm{e} 11$ & $\begin{array}{c}1.80 \mathrm{e}- \\
10\end{array}$ \\
$\begin{array}{c}\text { Condition } \\
3\end{array}$ & 0.163 & $\begin{array}{c}2.47 \mathrm{e} 1 \\
7\end{array}$ & $2.02 \mathrm{e}-17$ & 11.9 & $9.13 \mathrm{e} 1$ & $4.38 \mathrm{e}-$ & 9.27 & $2.78 \mathrm{e} 10$ & $\begin{array}{c}1.62 \mathrm{e}- \\
10\end{array}$ \\
$\begin{array}{c}\text { Condition } \\
4\end{array}$ & 0.170 & $\begin{array}{c}1.07 \mathrm{e} 1 \\
7\end{array}$ & $3.96 \mathrm{e}-17$ & 9.98 & $\begin{array}{c}2.96 \mathrm{e} 1 \\
2\end{array}$ & $\begin{array}{c}1.44 \mathrm{e}- \\
12\end{array}$ & 7.58 & $5.64 \mathrm{e} 10$ & $\begin{array}{c}7.53 \mathrm{e}- \\
11\end{array}$ \\
$\begin{array}{c}\text { Condition } \\
5\end{array}$ & 0.157 & $\begin{array}{c}5.28 \mathrm{e} 1 \\
7\end{array}$ & $1.09 \mathrm{e}-17$ & 12.4 & $\begin{array}{c}7.03 \mathrm{e} 1 \\
9\end{array}$ & $\begin{array}{c}8.18 \mathrm{e}- \\
12\end{array}$ & 9.62 & $2.44 \mathrm{e} 10$ & $\begin{array}{c}2.35 \mathrm{e}- \\
11\end{array}$ \\
$\begin{array}{c}\text { Condition } \\
6\end{array}$ & 0.154 & $\begin{array}{c}7.26 \mathrm{e} 1 \\
7\end{array}$ & $1.14 \mathrm{e}-17$ & 11.7 & $1.04 \mathrm{e} 1$ & $7.92 \mathrm{e}-$ & 8.70 & $3.48 \mathrm{e} 10$ & $2.37 \mathrm{e}-$ \\
10
\end{tabular}

Table 5. Fatigue Damage of Midspan Location when VC2 Vehicles Drive

\begin{tabular}{|c|c|c|c|c|c|c|c|c|c|}
\hline \multirow{2}{*}{$\begin{array}{l}\text { Working } \\
\text { condition }\end{array}$} & \multicolumn{3}{|c|}{ Concrete } & \multicolumn{3}{|c|}{ Reinforcement } & \multicolumn{3}{|c|}{ Prestressed reinforcement } \\
\hline & $\mathrm{S}_{\mathrm{eq}}$ & $\mathrm{N}$ & $\begin{array}{l}\text { Fatigue } \\
\text { damage }\end{array}$ & $\Delta \sigma_{\mathrm{eq}}$ & $\mathrm{N}$ & $\begin{array}{l}\text { Fatigue } \\
\text { damage }\end{array}$ & $\Delta \sigma_{\mathrm{eq}}$ & $\mathrm{N}$ & $\begin{array}{l}\text { Fatigue } \\
\text { damage }\end{array}$ \\
\hline $\begin{array}{c}\text { Condition } \\
1\end{array}$ & $\begin{array}{c}0.19 \\
6\end{array}$ & $\begin{array}{c}6.17 \mathrm{e} 1 \\
5\end{array}$ & $7.41 \mathrm{e}-16$ & $\begin{array}{c}10.9 \\
8\end{array}$ & $\begin{array}{c}1.60 \mathrm{e} 1 \\
2\end{array}$ & $\begin{array}{c}3.13 \mathrm{e}- \\
12\end{array}$ & 8.88 & $\begin{array}{c}3.24 \mathrm{e} 1 \\
0\end{array}$ & $1.39 \mathrm{e}-10$ \\
\hline $\begin{array}{c}\text { Condition } \\
2\end{array}$ & $\begin{array}{c}0.19 \\
7\end{array}$ & $\begin{array}{c}5.79 \mathrm{e} 1 \\
5\end{array}$ & $5.18 \mathrm{e}-16$ & $\begin{array}{c}12.0 \\
7\end{array}$ & $\begin{array}{c}8.75 \mathrm{e} 1 \\
1\end{array}$ & $\begin{array}{c}3.43 \mathrm{e}- \\
12\end{array}$ & 10.09 & $\begin{array}{c}2.07 \mathrm{e} 1 \\
0\end{array}$ & $1.45 \mathrm{e}-10$ \\
\hline $\begin{array}{c}\text { Condition } \\
3\end{array}$ & $\begin{array}{c}0.18 \\
0\end{array}$ & $\begin{array}{c}3.51 \mathrm{e} 1 \\
6\end{array}$ & $1.21 \mathrm{e}-16$ & $\begin{array}{c}15.0 \\
4\end{array}$ & $\begin{array}{c}2.15 \mathrm{e} 1 \\
1\end{array}$ & $\begin{array}{c}1.98 \mathrm{e}- \\
11\end{array}$ & 12.01 & $\begin{array}{c}1.15 \mathrm{e} 1 \\
0\end{array}$ & $3.90 \mathrm{e}-10$ \\
\hline $\begin{array}{c}\text { Condition } \\
4\end{array}$ & $\begin{array}{c}0.18 \\
2\end{array}$ & $\begin{array}{c}2.89 \mathrm{e} 1 \\
6\end{array}$ & $1.30 \mathrm{e}-16$ & $\begin{array}{c}12.3 \\
9\end{array}$ & $\begin{array}{c}7.43 \mathrm{e} 1 \\
1\end{array}$ & $\begin{array}{c}5.04 \mathrm{e}- \\
12\end{array}$ & 9.80 & $\begin{array}{c}2.29 \mathrm{e} 1 \\
0\end{array}$ & $1.64 \mathrm{e}-10$ \\
\hline $\begin{array}{c}\text { Condition } \\
5\end{array}$ & $\begin{array}{c}0.18 \\
3\end{array}$ & $\begin{array}{c}2.49 \mathrm{e} 1 \\
6\end{array}$ & $2.01 \mathrm{e}-16$ & $\begin{array}{c}13.1 \\
1\end{array}$ & $\begin{array}{c}5.17 \mathrm{e} 1 \\
1\end{array}$ & $\begin{array}{c}9.67 \mathrm{e}- \\
12\end{array}$ & 10.22 & $\begin{array}{c}1.97 \mathrm{e} 1 \\
0\end{array}$ & $2.53 \mathrm{e}-10$ \\
\hline $\begin{array}{c}\text { Condition } \\
6\end{array}$ & $\begin{array}{c}0.18 \\
3\end{array}$ & $\begin{array}{c}2.43 \mathrm{e} 1 \\
6\end{array}$ & $2.57 \mathrm{e}-16$ & $\begin{array}{c}12.3 \\
2\end{array}$ & $\begin{array}{c}7.69 \mathrm{e} 1 \\
1\end{array}$ & $\begin{array}{c}8.13 \mathrm{e}- \\
12\end{array}$ & 9.42 & $\begin{array}{c}2.63 \mathrm{e} 1 \\
0\end{array}$ & $2.37 \mathrm{e}-10$ \\
\hline
\end{tabular}
under Different Working Conditions 
Table 6. Fatigue Damage of Midspan Location when VC3 Vehicles Drive under Different Working Conditions

\begin{tabular}{|c|c|c|c|c|c|c|c|c|c|}
\hline \multirow{2}{*}{$\begin{array}{l}\text { Working } \\
\text { condition }\end{array}$} & \multicolumn{3}{|c|}{ Concrete } & \multicolumn{3}{|c|}{ Reinforcement } & \multicolumn{3}{|c|}{ Prestressed reinforcement } \\
\hline & $\mathrm{S}_{\mathrm{eq}}$ & $\mathrm{N}$ & $\begin{array}{l}\text { Fatigue } \\
\text { damage }\end{array}$ & $\Delta \sigma_{\mathrm{eq}}$ & $\mathrm{N}$ & $\begin{array}{l}\text { Fatigue } \\
\text { damage }\end{array}$ & $\Delta \sigma_{\mathrm{eq}}$ & $\mathrm{N}$ & $\begin{array}{l}\text { Fatigue } \\
\text { damage }\end{array}$ \\
\hline $\begin{array}{c}\text { Condition } \\
1\end{array}$ & $\begin{array}{c}0.10 \\
3\end{array}$ & $\begin{array}{c}2.33 \mathrm{e} 2 \\
1\end{array}$ & $1.18 \mathrm{e}-21$ & 17.22 & $\begin{array}{c}9.07 \mathrm{e} 1 \\
0\end{array}$ & $4.14 \mathrm{e}-11$ & $\begin{array}{c}14.8 \\
2\end{array}$ & $5.34 \mathrm{e} 9$ & $6.07 \mathrm{e}-10$ \\
\hline $\begin{array}{c}\text { Condition } \\
2\end{array}$ & $\begin{array}{c}0.15 \\
3\end{array}$ & $\begin{array}{c}8.99 \mathrm{e} 1 \\
7\end{array}$ & $2.78 \mathrm{e}-18$ & 17.62 & $\begin{array}{c}7.83 \mathrm{e} 1 \\
0\end{array}$ & $4.47 \mathrm{e}-11$ & $\begin{array}{c}14.7 \\
0\end{array}$ & $5.52 \mathrm{e} 9$ & $6.34 \mathrm{e}-10$ \\
\hline $\begin{array}{c}\text { Condition } \\
3\end{array}$ & $\begin{array}{c}0.22 \\
2\end{array}$ & $\begin{array}{c}5.56 \mathrm{e} 1 \\
4\end{array}$ & $6.75 \mathrm{e}-15$ & 19.16 & $\begin{array}{c}4.59 \mathrm{e} 1 \\
0\end{array}$ & $9.25 \mathrm{e}-11$ & $\begin{array}{c}14.8 \\
4\end{array}$ & $5.33 \mathrm{e} 9$ & $8.91 \mathrm{e}-10$ \\
\hline $\begin{array}{c}\text { Condition } \\
4\end{array}$ & $\begin{array}{c}0.21 \\
6\end{array}$ & $\begin{array}{c}9.62 \mathrm{e} 1 \\
4\end{array}$ & $3.38 \mathrm{e}-15$ & 17.34 & $\begin{array}{c}8.68 \mathrm{e} 1 \\
0\end{array}$ & $3.75 \mathrm{e}-11$ & $\begin{array}{c}14.3 \\
0\end{array}$ & $6.08 \mathrm{e} 9$ & $5.34 \mathrm{e}-10$ \\
\hline $\begin{array}{c}\text { Condition } \\
5\end{array}$ & $\begin{array}{c}0.20 \\
5\end{array}$ & $\begin{array}{c}2.57 \mathrm{e} 1 \\
5\end{array}$ & $1.27 \mathrm{e}-15$ & 18.75 & $\begin{array}{c}5.27 \mathrm{e} 1 \\
0\end{array}$ & $6.16 \mathrm{e}-11$ & $\begin{array}{c}15.0 \\
7\end{array}$ & $5.05 \mathrm{e} 9$ & $7.43 \mathrm{e}-10$ \\
\hline $\begin{array}{c}\text { Condition } \\
6\end{array}$ & $\begin{array}{c}0.20 \\
1\end{array}$ & $\begin{array}{c}4.04 \mathrm{e} 1 \\
5\end{array}$ & $1.42 \mathrm{e}-15$ & 17.38 & $\begin{array}{c}8.56 \mathrm{e} 1 \\
0\end{array}$ & $6.72 \mathrm{e}-11$ & $\begin{array}{c}13.5 \\
2\end{array}$ & $7.39 \mathrm{e} 9$ & $7.78 \mathrm{e}-10$ \\
\hline
\end{tabular}

Table 7. Fatigue Damage of Midspan Location when VC4 Vehicles Drive under Different Working Conditions

\begin{tabular}{|c|c|c|c|c|c|c|c|c|c|}
\hline \multirow[b]{2}{*}{$\begin{array}{l}\text { Working } \\
\text { condition }\end{array}$} & \multicolumn{3}{|c|}{ Concrete } & \multicolumn{3}{|c|}{ Reinforcement } & \multicolumn{3}{|c|}{ Prestressed reinforcement } \\
\hline & $\mathrm{S}_{\mathrm{eq}}$ & $\mathrm{N}$ & $\begin{array}{l}\text { Fatigue } \\
\text { damage }\end{array}$ & $\Delta \sigma_{\mathrm{eq}}$ & $\mathrm{N}$ & $\begin{array}{l}\text { Fatigue } \\
\text { damage }\end{array}$ & $\Delta \sigma_{\mathrm{eq}}$ & $\mathrm{N}$ & $\begin{array}{l}\text { Fatigue } \\
\text { damage }\end{array}$ \\
\hline $\begin{array}{c}\text { Condition } \\
1\end{array}$ & $\begin{array}{c}0.21 \\
2\end{array}$ & $\begin{array}{c}1.36 \mathrm{e} 1 \\
5\end{array}$ & $4.06 \mathrm{e}-15$ & 12.86 & $\begin{array}{c}5.84 \mathrm{e} 1 \\
1\end{array}$ & $1.03 \mathrm{e}-11$ & $\begin{array}{c}10.0 \\
5\end{array}$ & $\begin{array}{c}2.10 \mathrm{e} 1 \\
0\end{array}$ & $2.86 \mathrm{e}-10$ \\
\hline $\begin{array}{c}\text { Condition } \\
2\end{array}$ & $\begin{array}{c}0.21 \\
2\end{array}$ & $\begin{array}{c}1.34 \mathrm{e} 1 \\
5\end{array}$ & $4.85 \mathrm{e}-15$ & 13.25 & $\begin{array}{c}4.84 \mathrm{e} 1 \\
1\end{array}$ & $1.45 \mathrm{e}-11$ & $\begin{array}{c}10.1 \\
0\end{array}$ & $\begin{array}{c}2.06 \mathrm{e} 1 \\
0\end{array}$ & $3.39 \mathrm{e}-10$ \\
\hline $\begin{array}{c}\text { Condition } \\
3\end{array}$ & $\begin{array}{c}0.19 \\
9\end{array}$ & $\begin{array}{c}4.62 \mathrm{e} 1 \\
5\end{array}$ & $1.25 \mathrm{e}-15$ & 14.00 & $\begin{array}{c}3.39 \mathrm{e} 1 \\
1\end{array}$ & $2.14 \mathrm{e}-11$ & $\begin{array}{c}10.6 \\
0\end{array}$ & $\begin{array}{c}1.74 \mathrm{e} 1 \\
0\end{array}$ & $4.17 \mathrm{e}-10$ \\
\hline $\begin{array}{c}\text { Condition } \\
4\end{array}$ & $\begin{array}{c}0.20 \\
6\end{array}$ & $\begin{array}{c}2.48 \mathrm{e} 1 \\
5\end{array}$ & $2.52 \mathrm{e}-15$ & 12.88 & $\begin{array}{c}5.79 \mathrm{e} 1 \\
1\end{array}$ & $1.08 \mathrm{e}-11$ & 9.68 & $\begin{array}{c}2.39 \mathrm{e} 1 \\
0\end{array}$ & $2.82 \mathrm{e}-10$ \\
\hline $\begin{array}{c}\text { Condition } \\
5\end{array}$ & $\begin{array}{c}0.20 \\
0\end{array}$ & $\begin{array}{c}4.13 \mathrm{e} 1 \\
5\end{array}$ & $1.88 \mathrm{e}-15$ & 14.69 & $\begin{array}{c}2.51 \mathrm{e} 1 \\
1\end{array}$ & $3.29 \mathrm{e}-11$ & $\begin{array}{c}11.0 \\
5\end{array}$ & $\begin{array}{c}1.50 \mathrm{e} 1 \\
0\end{array}$ & $5.15 e-10$ \\
\hline $\begin{array}{c}\text { Condition } \\
6\end{array}$ & $\begin{array}{c}0.19 \\
8\end{array}$ & $\begin{array}{c}5.40 \mathrm{e} 1 \\
5\end{array}$ & $1.90 \mathrm{e}-15$ & 14.32 & $\begin{array}{c}2.95 \mathrm{e} 1 \\
1\end{array}$ & $3.48 \mathrm{e}-11$ & $\begin{array}{c}10.2 \\
9\end{array}$ & $\begin{array}{c}1.93 \mathrm{e} 1 \\
0\end{array}$ & $5.32 \mathrm{e}-10$ \\
\hline
\end{tabular}

Table 8. Fatigue Damage of Midspan Location when VC5 Vehicles Drive under Different Working Conditions

\begin{tabular}{|c|c|c|c|c|c|c|c|c|c|}
\hline \multirow[b]{2}{*}{$\begin{array}{l}\text { Working } \\
\text { condition }\end{array}$} & \multicolumn{3}{|c|}{ Concrete } & \multicolumn{3}{|c|}{ Reinforcement } & \multicolumn{3}{|c|}{ Prestressed reinforcement } \\
\hline & $\mathrm{S}_{\mathrm{eq}}$ & $\mathrm{N}$ & $\begin{array}{l}\text { Fatigue } \\
\text { damage }\end{array}$ & $\Delta \sigma_{\mathrm{eq}}$ & $\mathrm{N}$ & $\begin{array}{l}\text { Fatigue } \\
\text { damage }\end{array}$ & $\Delta \sigma_{\mathrm{eq}}$ & $\mathrm{N}$ & $\begin{array}{l}\text { Fatigue } \\
\text { damage }\end{array}$ \\
\hline $\begin{array}{c}\text { Condition } \\
1\end{array}$ & $\begin{array}{c}0.22 \\
5\end{array}$ & $\begin{array}{c}4.29 \mathrm{e} 1 \\
4\end{array}$ & $1.87 \mathrm{e}-14$ & 13.40 & $\begin{array}{c}4.50 \mathrm{e} 1 \\
1\end{array}$ & $2.00 \mathrm{e}-11$ & $\begin{array}{c}10.2 \\
2\end{array}$ & $\begin{array}{c}1.98 \mathrm{e} 1 \\
0\end{array}$ & $4.05 \mathrm{e}-10$ \\
\hline $\begin{array}{c}\text { Condition } \\
2\end{array}$ & $\begin{array}{c}0.22 \\
0\end{array}$ & $\begin{array}{c}6.41 \mathrm{e} 1 \\
4\end{array}$ & $1.17 \mathrm{e}-14$ & 13.78 & $\begin{array}{c}3.76 \mathrm{e} 1 \\
1\end{array}$ & $2.13 \mathrm{e}-11$ & $\begin{array}{c}10.2 \\
0\end{array}$ & $\begin{array}{c}1.99 \mathrm{e} 1 \\
0\end{array}$ & $4.02 \mathrm{e}-10$ \\
\hline $\begin{array}{c}\text { Condition } \\
3\end{array}$ & $\begin{array}{c}0.20 \\
5\end{array}$ & $\begin{array}{c}2.73 \mathrm{e} 1 \\
5\end{array}$ & $1.37 \mathrm{e}-15$ & 14.68 & $\begin{array}{c}2.52 \mathrm{e} 1 \\
1\end{array}$ & $2.28 \mathrm{e}-11$ & $\begin{array}{c}11.1 \\
8\end{array}$ & $\begin{array}{c}1.44 \mathrm{e} 1 \\
0\end{array}$ & $4.33 \mathrm{e}-10$ \\
\hline $\begin{array}{c}\text { Condition } \\
4\end{array}$ & $\begin{array}{c}0.21 \\
9\end{array}$ & $\begin{array}{c}7.06 \mathrm{e} 1 \\
4\end{array}$ & $8.50 \mathrm{e}-15$ & 13.67 & $\begin{array}{c}3.96 \mathrm{e} 1 \\
1\end{array}$ & $1.77 \mathrm{e}-11$ & $\begin{array}{c}10.1 \\
8\end{array}$ & $\begin{array}{c}2.01 \mathrm{e} 1 \\
0\end{array}$ & $3.49 \mathrm{e}-10$ \\
\hline $\begin{array}{c}\text { Condition } \\
5\end{array}$ & $\begin{array}{c}0.21 \\
8\end{array}$ & $\begin{array}{c}7.91 \mathrm{e} 1 \\
4\end{array}$ & $5.69 \mathrm{e}-15$ & 14.31 & $\begin{array}{c}2.95 \mathrm{e} 1 \\
1\end{array}$ & $2.37 \mathrm{e}-11$ & $\begin{array}{c}10.9 \\
9\end{array}$ & $\begin{array}{c}1.53 \mathrm{e} 1 \\
0\end{array}$ & $4.25 \mathrm{e}-10$ \\
\hline $\begin{array}{c}\text { Condition } \\
6\end{array}$ & $\begin{array}{c}0.21 \\
8\end{array}$ & $\begin{array}{c}7.47 \mathrm{e} 1 \\
4\end{array}$ & $1.07 \mathrm{e}-14$ & 13.76 & $\begin{array}{c}3.79 \mathrm{e} 1 \\
1\end{array}$ & $2.50 \mathrm{e}-11$ & 9.96 & $\begin{array}{c}2.16 \mathrm{e} 1 \\
0\end{array}$ & $4.40 \mathrm{e}-10$ \\
\hline
\end{tabular}


Calculated data in each table states that fatigue damage of concrete is the smallest but that of prestressed reinforcement is the largest under all conditions in the comparison among concrete, reinforcement and prestressed reinforcement. For concrete, its fatigue damage tends to increase when vehicles with 2-6 axles pass the bridge under the same working condition and the fatigue damage caused by 6 -axle vehicles for concrete is the largest.

As for regular reinforcement and prestressed reinforcement, the fatigue damage caused by 4 -axle vehicles is the largest under all working conditions. Besides, fatigue damage increases with 4-axle vehicle speed. Equivalent stress level of reinforcement and prestressed steel strand is lower than 20Mpa. When stress amplitude is less than $30 \mathrm{Mpa}$, fatigue life of twisted steel is a little longer than that of prestressed reinforcement under the same stress amplitude. When stress amplitude is larger than 30Mpa, fatigue life of prestressed steel strand is much longer than that of twisted steel with increase of stress amplitude, as showed in Figure 11. Accumulated fatigue damage is much smaller than 1 after the cycle has lasted 2 million times under each stress level. Thus, the bridge has adequate fatigue life under normal service period.

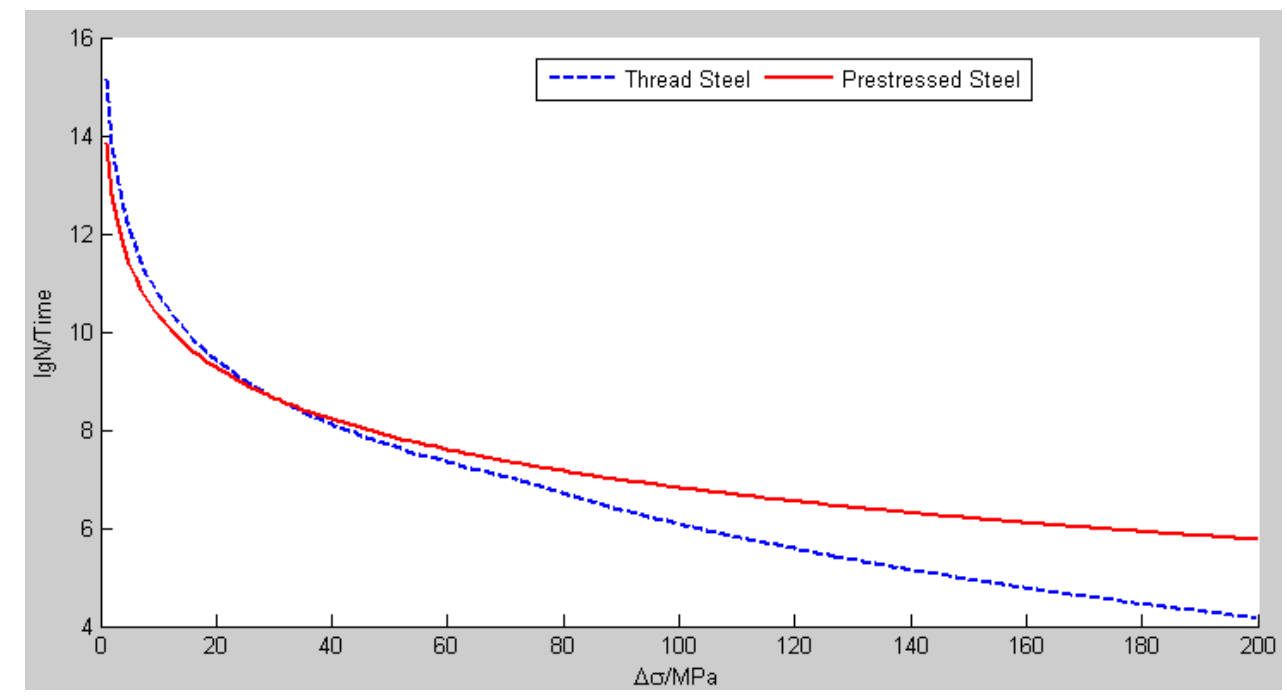

Figure 11. S-N Curves of Reinforcement

\subsection{Fatigue Damage under Random Vehicle Load}

4.2.1 A General Operation State: Random vehicle load sequence produced by MATLAB was loaded on the FEM. Meanwhile. The author extracted stress time-histories data about concrete, reinforcement and prestressed reinforcement in midspan pressure zones, performed counting by the rain-flow counting method and calculated fatigue of the bridge when random vehicles passed the bridge under a general stage. Random traffic flow simulated by this thesis under a general condition is that random vehicles on two lanes drive at $60 \mathrm{~km} / \mathrm{h}$. In detail, there are 50 random vehicles on each lane and 100 vehicles pass the bridge in all. By lateral distribution influence coefficient, random vehicle load was loaded on the FEM to do transient dynamic analysis. Stress time-history curves of midspan nodes and stress cycle statistics are showed in Figure 12 and Figure 13, respectively. 


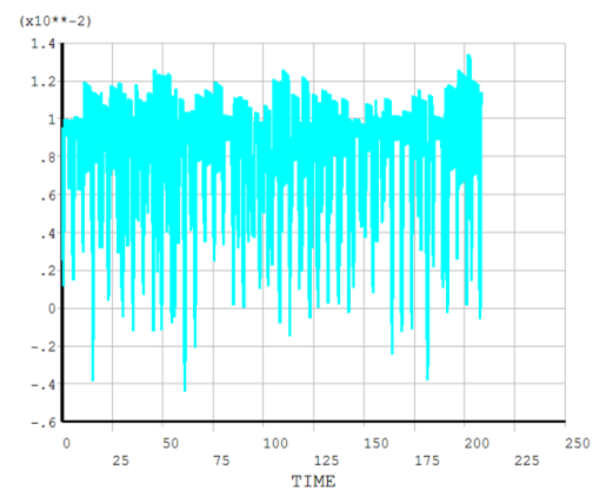

(a) Vertical displacement

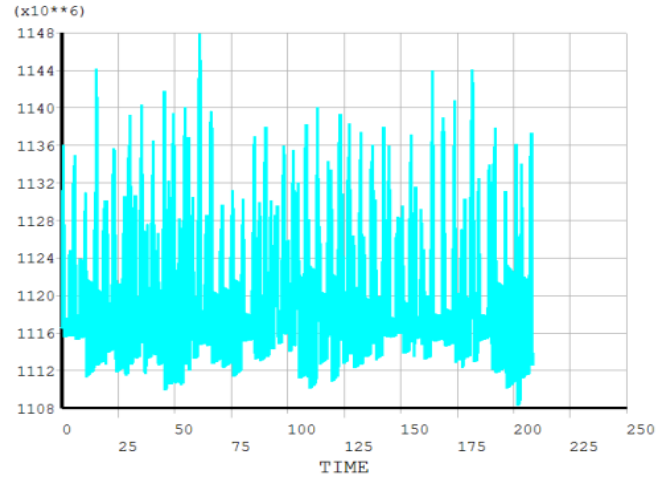

(c) Axial stress of prestressed reinforcement

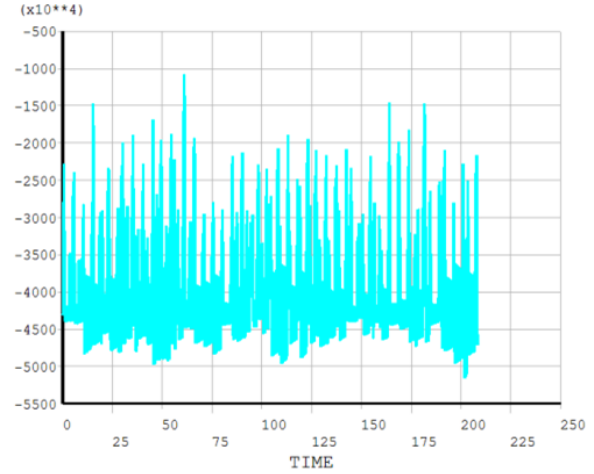

(b) Axial stress of regular reinforcement

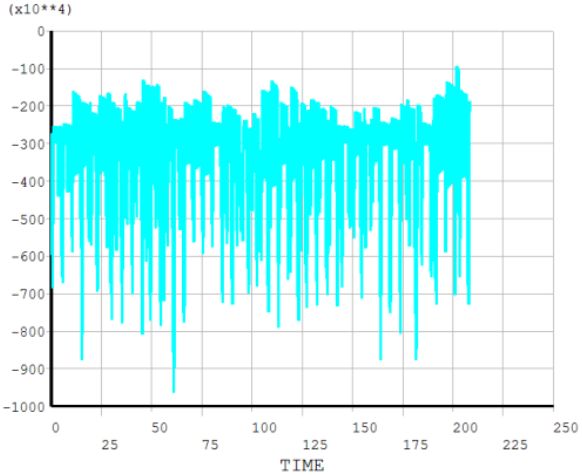

(d) Concrete stress in pressure zones

Figure 12. Time-history Curves of Midspan Nodes under a General Operation State

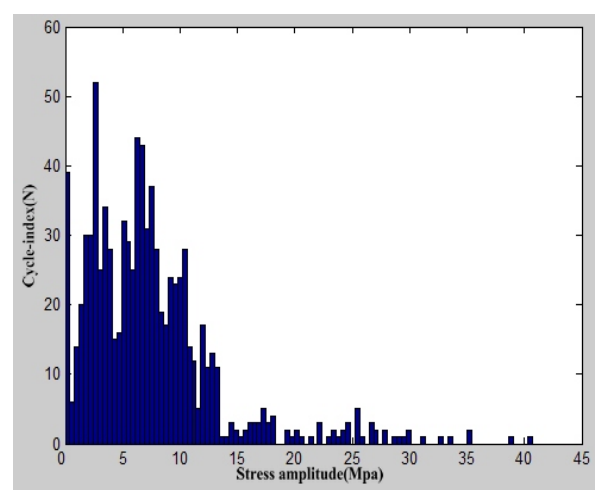

(a) $12 \mathrm{~mm}$ reinforcement

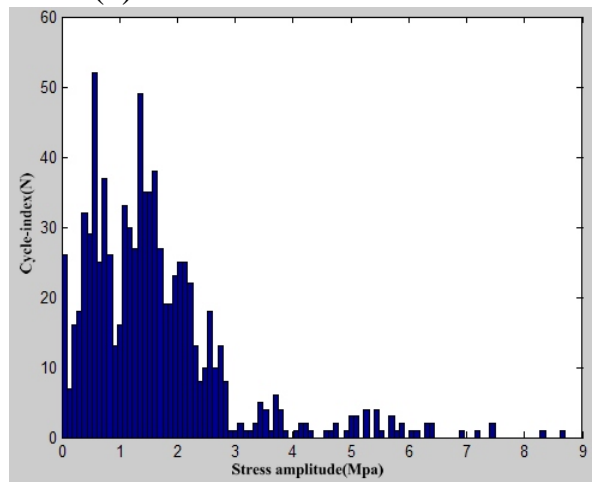

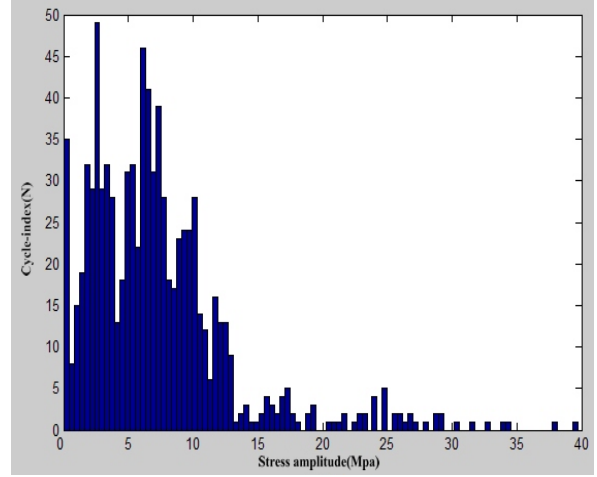

(b) Prestressed reinforcement

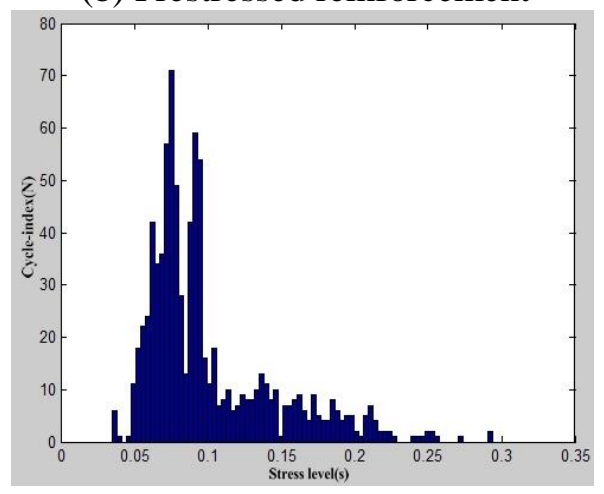

(c) Concrete in the pressure zone

Figure 13. Stress Cycle Statistics of Midspan Nodes at the Bridge under a General Operation State 
From above results, under a general operation state, stress amplitude of both reinforcement and prestressed steel strand is below $15 \mathrm{Mpa}$ and that of concrete is below $3 \mathrm{Mpa}$. Besides, stress level mainly concentrates on $0.06 \sim 0.2$. Formulas 1.8 and 1.9 show that under cyclic action of $15 \mathrm{Mpa}$ stress amplitude, fatigue life $(\mathrm{N})$ of reinforcement is greater than $2.19 * 1011$ and prestressed reinforcement is greater than 5.13*109, respectively. In addition, fatigue life $(\mathrm{N})$ of concrete in the pressure zone is greater than $4.26 * 1017$ under 0.2 stress level. Thus, the structure has adequate fatigue life.

4.2.2 An Intensive Operation State: For comparison, random traffic flow produced by MATLAB under an intensive condition also simulated two lanes with 50 random vehicles for each lane. Lateral loading position was as under a general operation state. The intensive operation state usually appears when there is a traffic jam. Distance headway is shorter and vehicle speed is lower in this case. Thus a speed of $20 \mathrm{~km} / \mathrm{h}$ was used to pass the bridge. Figure 14 shows time-history curves that correspond to the midspan position under an intensive operation state. And Figure 15 indicates the stress cycle statistics after applying the rain-flow counting method.

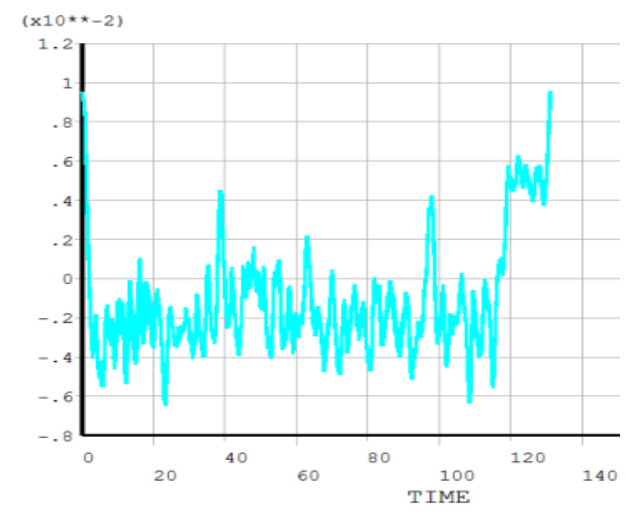

(a) Vertical displacement

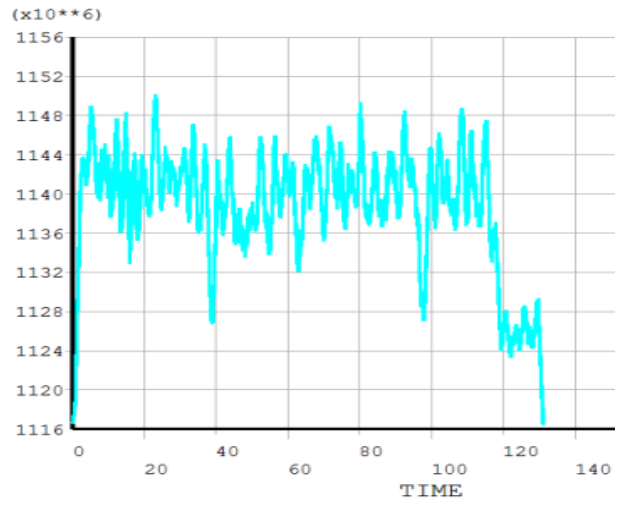

(c) Axial stress of prestressed reinforcement

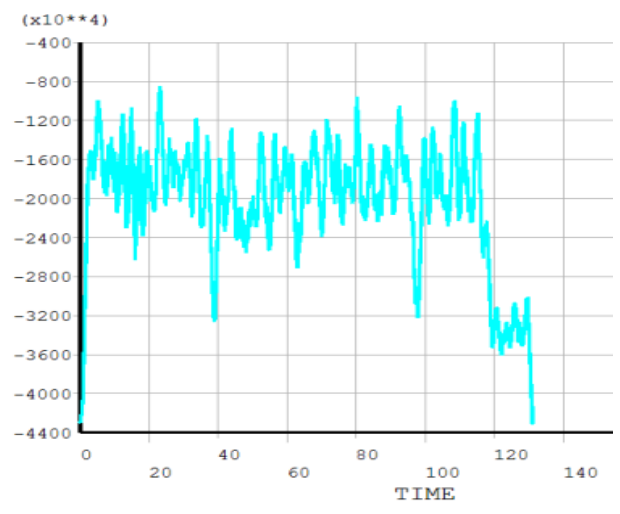

(b) Axial stress of regular reinforcement

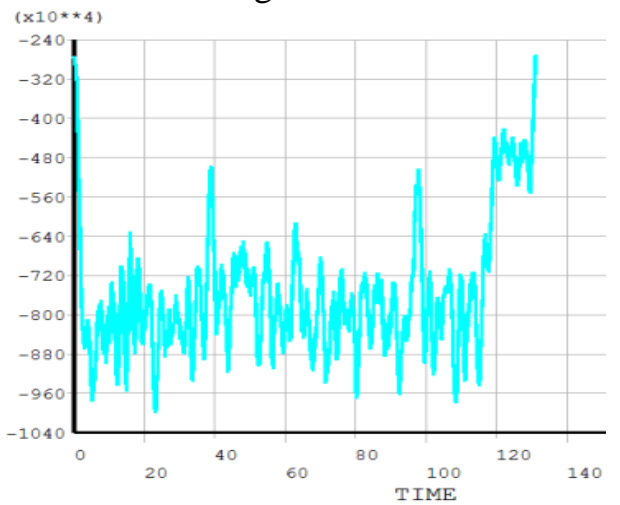

(d) Concrete stress in pressure zones

Figure 14. Time-history Curves of Midspan Nodes under an Intensive Operation State 


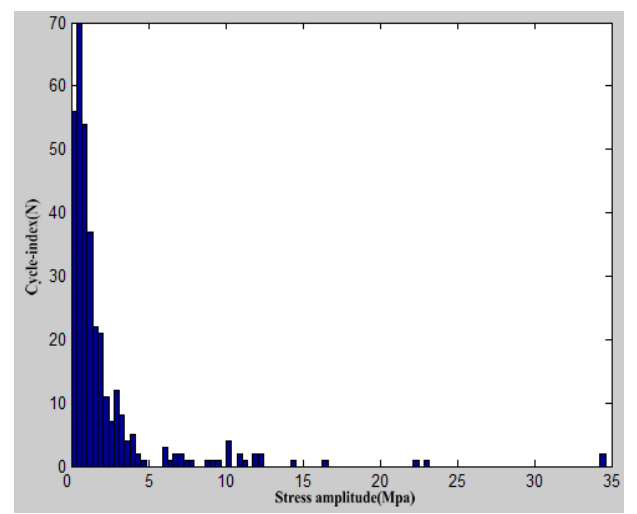

(a) $12 \mathrm{~mm}$ reinforcement

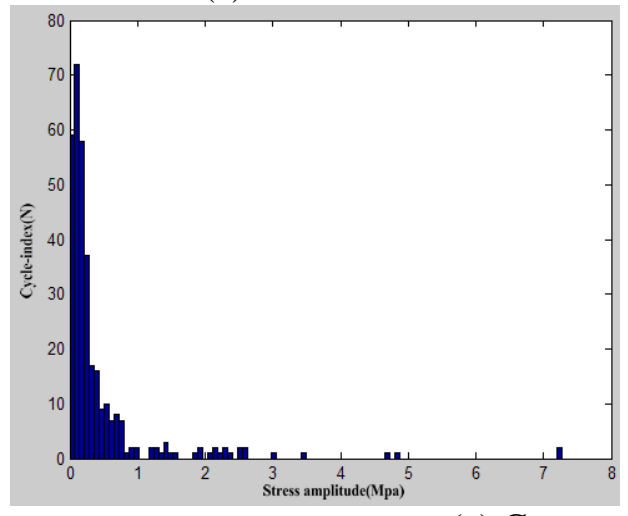

(c) Concrete in the pressure zone

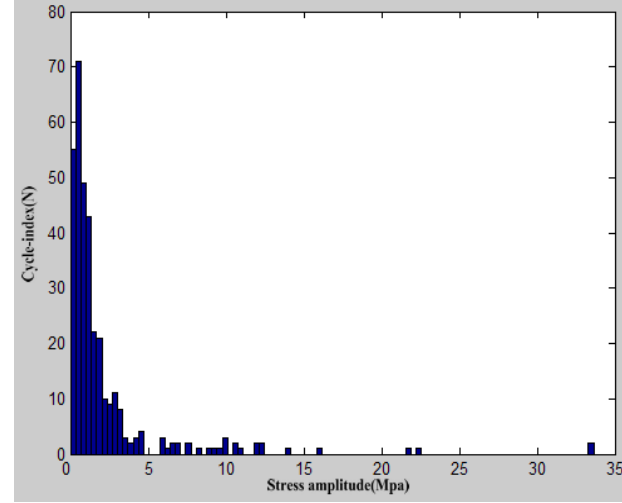

(b) Prestressed reinforcement

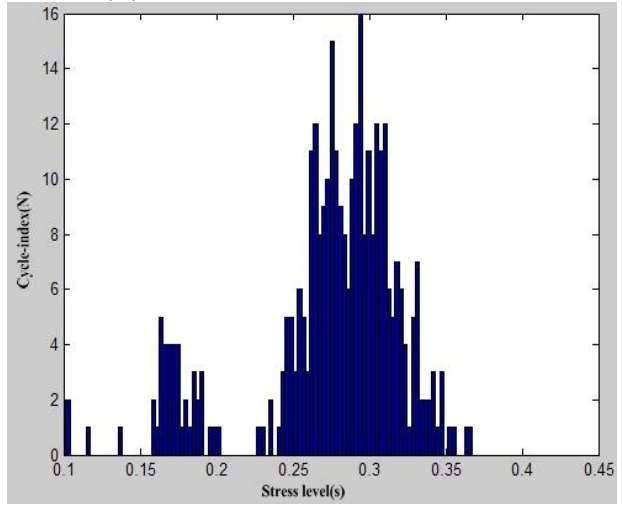

\section{Figure 15. Stress Cycle Statistics of Midspan Nodes at the Bridge under an Intensive Operation State}

Calculated results under an intensive operation state tell that most stress amplitude of reinforcement and prestressed reinforcement is less than $5 \mathrm{Mpa}$ and stress amplitude for concrete is $1 \mathrm{Mpa}$. They are all about $2 / 3$ time lower than that under a general operation state. Stress level of concrete in the pressure zone is mainly between 0.25 and 0.35 , which is greater than that under a general operation state. When $S=0.3$, the corresponding fatigue life is $\mathrm{N}=1.369 * 1012$, being 0.4 million times smaller than that under a general operation state.

Table 9 lists the summary data for the two conditions. With respect to the twisted steel and prestressed reinforcement, the fatigue damage under a general operation state is 9.91 times and 12.55 times higher than that under an intensive operation state, respectively. The fatigue damage caused by vehicles for the bridge is smaller under an intensive operation state

\section{Table 9. Fatigue Damage at Midspan Position of the Bridge under Effect of Random Traffic Flow}

\begin{tabular}{cccccccccc}
\hline \multirow{2}{*}{$\begin{array}{c}\text { Operation } \\
\text { state }\end{array}$} & $\mathrm{S}_{\mathrm{eq}}$ & $\mathrm{N}$ & $\begin{array}{c}\text { Fatigue } \\
\text { damage }\end{array}$ & $\Delta \sigma_{\mathrm{eq}}$ & $\mathrm{N}$ & $\begin{array}{c}\text { Fatigue } \\
\text { damag } \\
\mathrm{e}\end{array}$ & $\Delta \sigma_{\mathrm{eq}}$ & $\mathrm{N}$ & $\begin{array}{c}\text { Fatigue } \\
\text { damage }\end{array}$ \\
\hline $\begin{array}{c}\text { A general } \\
\text { operation } \\
\text { state }\end{array}$ & $\begin{array}{c}0.21 \\
9\end{array}$ & $\begin{array}{c}6.91 \mathrm{e} 1 \\
4\end{array}$ & $\begin{array}{c}1.23 \mathrm{e}- \\
12\end{array}$ & $\begin{array}{c}17.5 \\
2\end{array}$ & $8.12 \mathrm{e} 10$ & $\begin{array}{c}1.07 \mathrm{e}- \\
8\end{array}$ & 12.03 & $\begin{array}{c}1.11 \mathrm{e} 1 \\
0\end{array}$ & $7.77 \mathrm{e}-8$ \\
\hline $\begin{array}{c}\text { An intensive } \\
\text { operation } \\
\text { state }\end{array}$ & $\begin{array}{c}0.31 \\
0\end{array}$ & $\begin{array}{c}7.24 \mathrm{e} 1 \\
1\end{array}$ & $\begin{array}{c}4.61 \mathrm{e}- \\
10\end{array}$ & $\begin{array}{c}14.1 \\
8\end{array}$ & $3.13 \mathrm{e} 11$ & $\begin{array}{c}1.08 \mathrm{e}- \\
9\end{array}$ & 7.64 & $\begin{array}{c}5.49 \mathrm{e} 1 \\
0\end{array}$ & $6.19 \mathrm{e}-9$ \\
\hline
\end{tabular}


The reason is belonging to this scenario vehicle speed is low and equivalent stress amplitude caused to the bridge is high when vehicles drive at a higher speed. If 12,000 vehicles pass the bridge averagely each day, fatigue life $(\mathrm{Y})$ of the bridge under a general operation state will be 139 years. Thus, under the current operational load condition, we can provide the bridge will not get fatigue failure.

\subsection{Fatigue Damage under Effect of Standard Fatigue Vehicle Models}

By consulting the way to load standard fatigue vehicle models in British specifications [3], we put the standard fatigue deduced vehicle model (Figure 3.3) and Liaoning standard fatigue vehicle models [11] into the same speed for comparison. Loaded them on the model at $60 \mathrm{~km} / \mathrm{h}$ for fatigue analysis. Results are indicated in Tables 10-11.

\section{Table 10. Fatigue Damage at the Midspan Position of the Bridge under Effect of Fatigue Vehicle Models}

\begin{tabular}{|c|c|c|c|c|c|c|c|c|c|}
\hline \multirow{2}{*}{$\begin{array}{c}\text { Acting } \\
\text { working } \\
\text { condition }\end{array}$} & \multicolumn{3}{|c|}{ Concrete } & \multicolumn{3}{|c|}{ Reinforcement } & \multicolumn{3}{|c|}{$\begin{array}{l}\text { Prestressed } \\
\text { reinforcement }\end{array}$} \\
\hline & $\mathrm{S}_{\mathrm{eq}}$ & $\mathrm{N}$ & $\begin{array}{l}\text { Fatigue } \\
\text { damage }\end{array}$ & $\Delta \sigma_{\mathrm{eq}}$ & $\mathrm{N}$ & $\begin{array}{l}\text { Fatigue } \\
\text { damage }\end{array}$ & $\Delta \sigma_{\mathrm{eq}}$ & $\mathrm{N}$ & $\begin{array}{l}\text { Fatigue } \\
\text { damage }\end{array}$ \\
\hline $\begin{array}{l}\text { Standard } \\
\text { Fatigue } \\
\text { vehicle } \\
\text { models }\end{array}$ & $\begin{array}{c}0.31 \\
8\end{array}$ & $\begin{array}{c}4.37 \mathrm{e} 1 \\
1\end{array}$ & $2.01 \mathrm{e}-11$ & $\begin{array}{c}16.9 \\
2\end{array}$ & $\begin{array}{c}1.02 \mathrm{e} 1 \\
1\end{array}$ & $9.43 e-11$ & 12.50 & $\begin{array}{c}9.73 \mathrm{e} \\
9\end{array}$ & $\begin{array}{c}9.45 \mathrm{e}- \\
10\end{array}$ \\
\hline $\begin{array}{c}\text { Liaoning } \\
\text { standard } \\
\text { fatigue } \\
\text { vehicle } \\
\text { models }\end{array}$ & $\begin{array}{c}0.31 \\
4\end{array}$ & $\begin{array}{c}5.55 \mathrm{e} 1 \\
1\end{array}$ & $1.66 \mathrm{e}-11$ & $\begin{array}{c}16.8 \\
6\end{array}$ & $\begin{array}{c}1.04 \mathrm{e} 1 \\
1\end{array}$ & $9.82 \mathrm{e}-11$ & 12.32 & $\begin{array}{c}10.3 \mathrm{e} \\
9\end{array}$ & $\begin{array}{c}9.75 \mathrm{e}- \\
10\end{array}$ \\
\hline
\end{tabular}

\section{Table 11. Comparison about Equivalent Stress Amplitude under Effect of} Fatigue Vehicles and Practical Running Vehicles

\begin{tabular}{ccccc}
\hline & \multicolumn{2}{c}{ Reinforcement } & Prestressed reinforcement \\
\cline { 2 - 5 } Vehicle load effect & $\Delta \sigma_{\mathrm{eq}}$ & $\begin{array}{c}\text { Ratio to equivalent stress } \\
\text { amplitude of practical } \\
\text { traffic flow }\end{array}$ & $\Delta \sigma_{\mathrm{eq}}$ & $\begin{array}{c}\text { Ratio to equivalent } \\
\text { stress amplitude of } \\
\text { practical traffic flow }\end{array}$ \\
\hline $\begin{array}{c}\text { A general operation } \\
\text { condition }\end{array}$ & 17.52 & 1 & 12.03 & 1 \\
\hline $\begin{array}{c}\text { An intensive } \\
\text { operation condition }\end{array}$ & 16.92 & 0.97 & 12.50 & 1.04 \\
\hline $\begin{array}{c}\text { Standard fatigue } \\
\text { vehicles on a single } \\
\text { lane }\end{array}$ & 10.38 & 0.59 & 7.67 & 0.64 \\
\hline $\begin{array}{c}\text { Liaoning standard } \\
\text { fatigue vehicle } \\
\text { models }\end{array}$ & 16.86 & 0.96 & 12.32 & 1.02 \\
\hline
\end{tabular}

Comparative results indicate equivalent stress amplitude caused by the fatigue vehicles loads assigned in this study is next to that under a general operation state. If we follow the situation that standard fatigue vehicles are loaded on a single lane in British specification [3], the ratio was 0.59 , which was lower than 0.97 . Thus, we may know that the loading method in this thesis considers vehicle situations on different lanes, which are more practical. Meanwhile, it is consistent with the fatigue effect that has been proposed in Literature [11] and that is appropriate for highway bridges in China under standard 
fatigue vehicles. Therefore, we could conclude the model proposed in this thesis was reasonable and may be used further for fatigue analysis.

\section{Conclusion}

1) Vehicles on the road bridge have strong randomness. Vehicle type, speed, distance, load and so are constantly evolving over time. Research on random load is still insufficient. In this paper, manual recording, live cameras, vehicle dynamic weighing system(WIM) were used to conduct a traffic investigation for 24 hours continuously. Via these surveys, typical vehicle load spectrum was established. After that, we used MATLAB to compile a random vehicle load generating program and simulated practical operational states of the bridge. The results show that the MATLAB programming with parameter control has universality.

2) The paper used a separation method to establish FEM and realized loading of produced random traffic flow on the model. Besides, we adopted Transient dynamic analysis method was adopted to get solutions, extracted stress timehistory and analyzed fatigue damage. Such a simulation is more realistic. The comparison between calculated results under different working conditions of typical vehicle models demonstrates that dynamic response amplitude of the bridge increases as vehicle speed grows. Impacts of different headway distance are minor for vehicles with 2-5 axles. Considering 6-axle vehicles, influence of changes in both vehicle speed and the headway distance is inconsiderable.

3) The comparison between a general operational state and an intensive operation state shows equivalent stress amplitude and fatigue damage under the latter condition are relatively smaller. By calculating fatigue damage and life of the bridge without influence of environmental factors, we may believe that fatigue failure won' t occur under a normal operation state.

4) Pass the contrast, verify the standard fatigue vehicle model in this thesis may act as reference for fatigue computations.

5) All the work above can facilitate examination of in-service bridges, calculation and prediction of their fatigue life. Also it can provide reference for perfecting bridge load design specifications.

\section{References}

[1] Z.-h. Xia, Z.-h. Zong and J.-w. Li, "Moving load identification of long span continuous rigid frame bridges based on health monitoring system", China Journal of Highway and Transport, (2012), vol. 25, no. 5, pp. 95-104.

[2] Y. Feng, R. Xiao-song and Z. Jin-cheng, "Progress in fatigue loading for highway steel bridges", Progress in Steel Building Structures, (2009), vol. 11, no. 2, pp. 35-39.

[3] British Standards Institution: Steel, Concrete and Composite Bridges-Part 10: Code of Practice for Fatigue, BS5400, (2002).

[4] American Association of State Highway and Transportation Officials. AASHTO LRFD Bridge Design Specifications, (2005)

[5] Code for design of highway bridges. Japan Road Association, (2002).

[6] P. Pokorný, L. Náhlík and P. Hutař, "Comparison of Different Load Spectra on Residual Fatigue Lifetime of Railway Axle”, Procedia Engineering, vol.74, (2014), pp. 313-316.

[7] S. Mikheevskiy, G. Glinka and E. Lee, "Fatigue Crack Growth Analysis Under Spectrum Loading in Various Environmental Conditions", Metallurgical and Materials Transactions, vol. 44, no. 3, (2013), pp. $1301-1310$

[8] J. J. Jensen, "Extreme value predictions using Monte Carlo simulations with artificially increased load spectrum", Probabilistic Engineering Mechanics, vol. 26, no. 2, (2010), pp. 399-404.

[9] B. Enright, C. Carey and C. C. Caprani, "Microsimulation Evaluation of Eurocode Load Model for American Long-Span Bridges", Journal of Bridge Engineering, ASCE (2013), vol. 18, no. 12, pp. 12521260.

[10] T. Lewei, S. Zuyan and C. Zhongyan, "Extreme value predictions using Monte Carlo simulations with artificially increased load spectrum”, China Civil Engineering Journal, vol. 30, no. 5, (1997), pp. 20-27. 
[11] Y. Zhou, W. Bao, H. Zhai and Y. Liu, "Research on fatigue design load standards of highway steel bridges", Journal of China Civil Engineering, vol. 43, no. 11, (2010), pp. 79-85.

[12] H.-b. Zhu, "Method and experiment research on highway reinforced concrete simply-supported girder bridge's fatigue residual service life forecast", Central South University, Changsha (2011).

[13] A. J. Kappos, I. G. Gidaris and K. I. Gkatzogias, "Problems associated with direct displacement-based design of concrete bridges with single-column piers, and some suggested improvements", Bulletin of Earthquake Engineering, vol. 10, no. 4, (2012), pp. 1237-1266.

[14] J.-M. Battini and M. Ülker-Kaustell, "A simple finite element to consider the non-linear influence of the ballast on vibrations of railway bridges", Engineering Structures, vol. 33, no. 9, (2011), pp. 2597-2602.

[15] D. B. Ashebo, T. H. T. Chan and L. Yu, "Evaluation of dynamic loads on a skew box girder continuous bridge Part II : Parametric study and dynamic load factor”, Engineering Structures, vol. 29, no. 6, (2007), pp. 1064-1073.

[16] P. Croce and W. Salvatore, "Stochastic Model for Multilane Traffic Effects on Bridges", Journal of Bridge Engineering, ASCE, vol. 6, no. 2, (2001), pp. 136-143.

[17] A. Turer, B. M. Shahrooz, "Load rating of concrete-deck-on-steel-stringer bridges using field-calibrated 2D-grid models", Engineering Structures, vol. 33, no. 4, (2011), pp. 1267-1276.

[18] L. Frýba and M. Pirner, "Load tests and modal analysis of bridges", Engineering Structures, vol. 23, no. 1, (2001), pp. 102-109.

[19] D. Markulak and B. Androić, "Modeling actual traffic load on road bridges", Građevinar, vol. 55, no. 3, (2003), pp. 129-135.

[20] X. Li, W. Ren and J. Zhong, "Research on standard fatigue vehicle load of highway bridges in southwestern mountainous areas", Journal of Vibration and Shock, vol. 31, no. 15, (2012), pp. 96-100.

[21] H. Huang and M. Liu, "Analysis about dynamic performance of reinforced concrete bridges under effect of vehicle load spectrum”, Chang'an University, Xi'an (2005).

[22] Miao T J, Chan T H T, "Bridge live load models from WIM data", Engineering Structure, vol. 24, no. 8, (2002), pp. 1071-1084.

[23] G. K. Fu and O. Hag-Elsafi, "Vehicular Overloads: Load Model, Bridge Safety, and Permit Checking", Journal of Bridge Engineering, ASCE, vol. 5, no. 1, (2000), pp.49-57.

[24] Y. Li and H. Che, "Research on flexural fatigue accumulated damage performance of concrete", China Railway Science, vol. 19, no. 2, (1998), pp. 52-59.

[25] Y. Li and H. Che, "Research on flexural fatigue performance of concrete under effect of equiamplitude repetitive stress", Journal of the China Railway Society, vol. 21, no. 2, (1999), pp.76-79.

[26] Y. J. Kim, "Safety assessment of steel-plate girder bridges subjected to military load classification", Engineering Structures, vol. 38, (2012), pp. 21-31.

[27] X. W. Ye, Y. Q. Ni and K. Y. Wong, "Statistical analysis of stress spectra for fatigue life assessment of steel bridges with structural health monitoring data", Engineering Structures, vol. 45, (2012), pp. 166176.

[28] K.-Y. Park and G.-M. Park, "A study on intelligent video security surveillance system with active tracking technology in multiple objects environment", International Journal of Security and Its Application, vol. 6, no. 2, (2012), pp. 211-216.

[29] J. R. Casas and J. D. Gómez, "Load rating of highway bridges by proof-loading”, KSCE Journal of Civil Engineering, vol. 17, no. 3, (2013), pp. 556-567.

[30] N. R. Mead, V. Viswanathan and J. Zhan, "Incorporating security requirements engineering into standard lifecycle processes", International Journal of Security and Its Application, vol. 2, no. 4, (2008), pp. 6780.

[31] J. C. Davis, "Live-load models for design and fatigue evaluation of highway bridges", Rutgers The State University of New Jersey - New Brunswick, New Brunswick (2007).

[32] M. Darbari, S. Medhavi and A. K. Srivastava, "Development of effective urban road traffic management using workflow techniques for upcoming metro cities like Lucknow(India) ", International Journal of Security and Its Application, vol. 2, no. 2, (2008), pp. 87-96.

\section{Authors}

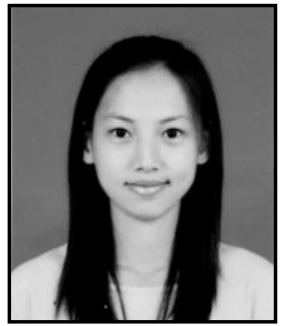

Xin Qin, She was born in 1987, Baoding, Hebei province. In 2011, she completed undergraduate work and received Bachelor of Engineering. Now she is a Ph.D. in Hebei University of Technology, Tianjin. Her major research is "Heavy traffic analysis software". And she wants to get the Ph.D. degree in May or June of 2016. 


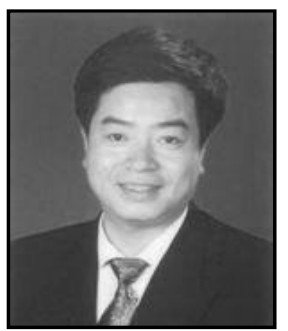

Dou Yuanming, He was born in 1956. Now he is a Professor and $\mathrm{PhD}$ supervisor in the School of Civil Engineering, Hebei University of Technology, Tianjin. Also he is the commissioner of State-owned Assets. He obtained his $\mathrm{PhD}$ in Civil Engineering from the TIANJIN University in 2002. His research work has centered on teaching and researching on building materials and structural engineering. He is a Professor and $\mathrm{PhD}$ supervisor in the School of Civil Engineering, Hebei University of Technology, Tianjin. He obtained his $\mathrm{PhD}$ in Civil Engineering from the TIANJIN University in 2002. His research work has 佛focused on teaching and research on building materials and structural engineering

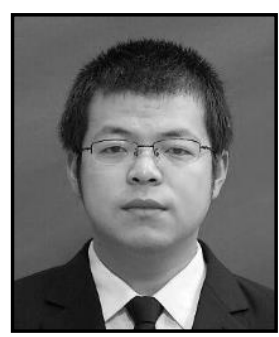

Chen Wan, He was born in 1989, Shanxi province. He just graduated from Hebei university of Technology in Jan. 2015. Before joining Hebei University of Technology, he was a master student of Tianjin university of Technology, and got his master degree in 2011. His major research is bridge structure stability. 
International Journal of Security and Its Applications

Vol.9, No.7 (2015) 